\title{
Adolescentes en trabajos peligrosos: análisis bajo un enfoque intergeneracional
}

\author{
Adolescents in hazardous work: analysis \\ under an intergenerational approach
}

Juan Francisco Suárez Martínez ${ }^{1}$ y Cinthya Guadalupe Caamal Olvera²

Fecha de recepción: 2 de marzo de 2021

Fecha de aceptación: 19 de mayo de 2021

1 - Nacionalidad: Mexicana. Grado: Doctorado en Ciencias Económicas. Adscripción: Universidad de Monterrey (D) ORCID: https://orcid.org/0000-0002-6256-0551 | Correo electrónico: juan.suarez@udem.edu

2 - Nacionalidad: Mexicana. Grado: Doctorado en Economía. Adscripción: Universidad Autónoma de Nuevo León (DD ORCID: https://orcid.org/0000-0003-0249-4027 | Correo electrónico: cinthya.caamal@gmail.com 


\section{Resumen}

El artículo analiza el efecto intergeneracional de la ocupación de los padres a los hijos adolescentes. La hipótesis es probar si la actividad económica de los padres influye en la probabilidad de que los jóvenes de 12 a 17 años se ocupen en trabajos peligrosos. Se propone una medida para cuantificar el trabajo peligroso multidimensional (TPM), cuyas estimaciones indican que el $31 \%$ de los adolescentes ocupados están en un TPM. Los resultados evidencian que, si los adolescentes trabajan en el mismo sector económico del padre, la probabilidad de que estén ocupados en un TPM aumenta en 10.3 puntos porcentuales ( $p p$ ). En contraste, si están en la misma actividad económica que la madre, se reduce entre 5.5 y 6.4 pp.

Palabras clave: trabajo peligroso, adolescentes, efecto intergeneracional, actividad económica.

\section{Abstract}

The article analyzes the intergenerational effect of the parents' occupation towards their adolescent children who work. The hypothesis is to test whether the parents' economic activity influences the probability that adolescents between 12 and 17 years old will engage in dangerous jobs. We developed a measure to quantify multidimensional hazardous work (TPM), whose estimates indicate that $31 \%$ of employed adolescents are in TPM. Results show that if adolescents work in the same economic sector as their father, their probability of being employed in a TPM increases by 10.3 percentage points ( $p p$ ). In contrast, if it is the same economic activity as the mother, it reduces between 5.5 and 6.4 pp.

Keywords: hazardous work, adolescents, intergenerational effect, economic activity. 


\section{Introducción}

$\mathrm{E}$ lobjetivo de este artículo es analizar la transferencia intergeneracional de la actividad económica entre padres a hijos adolescentes. Si los jóvenes ingresan al mercado laboral como ayudantes, ya sea en la ocupación del padre o de la madre, es altamente probable que ese empleo sea el mismo que alguno de sus padres, al menos en el primer empleo. Entonces, la ocupación de los padres podría ser un buen predictor de la ocupación en la cual se desarrollará el adolescente. El sector económico en el que se encuentran trabajando los padres podría impactar en el tipo de trabajo de los adolescentes ya que, si el padre/madre trabajan en un lugar peligroso podría poner en riesgo la salud física y/o mental de los adolescentes (Puga y Solís, 2010; Doruk et al., 2020).

Según la Organización Internacional del Trabajo (OIT) un trabajo peligroso es cuando los jóvenes están expuestos a una sustancia tóxica, a una actividad desgastante, a un aparato peligroso o a una situación que les genere estrés, es decir, un trabajo que tiene el potencial de causar enfermedades, daños cerebrales o lesiones físicas; o que dificulta el desarrollo emocional, intelectual o físico del adolescente (OIT, 2018). Bajo esta óptica, se estima en el mundo que 73 millones de los niños de 5 a 17 años están ocupados en trabajos peligrosos, prevalencia estimada en 4.7\% para el año 2017 (OIT, 2018). Un mayor porcentaje de hombres de 5 a 17 años está realizando trabajos peligrosos, $5.5 \%$, y el 3.6\% de las mujeres. En el continente africano es en donde se estima la mayor prevalencia de trabajo peligroso, de $8.6 \%$, mientras que las otras regiones tienen una prevalencia menor, en Europa y Asia Central es de 4\%, en Asia y El Pacífico de 3.4\% y en los países del continente americano es de 3.2\%. Por grupos de edad, se encuentra una menor incidencia en los menores de 5 a 14 años, estimada en $2.9 \%$, y una mayor prevalencia del trabajo peligroso en los adolescentes de 15 a 17 años, $10.5 \%$ (OIT, 2018).

Los datos del Módulo de Trabajo Infantil (MTI), INEGI (2017b), indican que 3.2 millones de niños de 5 a 17 años se encuentran trabajando, lo que, en términos porcentuales es de $10.9 \%$. Entonces, es importante analizar qué proporción de estos adolescentes se encuentran ocupados en trabajos peligrosos y cuáles son las razones que los llevan a caer en este tipo de trabajos, ya que a esta edad aún se encuentran en una etapa de crecimiento y desarrollo; además, al tener menos experiencia son más vulnerables a ser explotados, y que sufran alguna enfermedad o lesión en comparación con los adultos (OIT, 2018). De acuerdo con INEGI (2018) la ocupación peligrosa, medida de acuerdo con la Ley Federal de Trabajo (LFT), es en donde laboran los menores en ocupaciones y sectores peligrosos que ponen en riesgo la salud y sano desarrollo, además de que laboran en horarios prolongados o nocturnos (Secretaría del Trabajo y Previsión Social, 2019). La cifra estimada para el 2017, en México, es de 3.6\% de los niños de 5 a 14 años, y de 18.3\% en los adolescentes de 15 a 17 años. Estas cifras reflejan que el trabajo peligroso en México es superior al promedio mundial estimado, entre los adolescentes de 15 a 17 años.

En México, las condiciones laborales permitidas para los trabajadores menores de edad se rigen de acuerdo con la LFT, por lo que, se podría calcular el porcentaje de menores que no cumplen con alguna de las condiciones de ley, como los resultados de INEGI (2018). El problema es que son varias 
condiciones estipuladas en la ley, lo que nos lleva a tomar decisiones sobre cuántas, cuáles y cómo ponderar cada una de las condiciones no permitidas en la LFT y poder estimar el porcentaje de menores en trabajos peligrosos. Por una parte, una medida unidimensional, que considere de forma independiente las condiciones laborales no adecuadas, podría sobreestimar el porcentaje de menores en trabajos peligrosos. Por otra parte, una medida que imponga excesivas condiciones de incumplimiento generaría un porcentaje subestimado, y que aportaría poca información sobre la realidad de las condiciones laborales de los adolescentes. No existe una metodología normalizada para determinar qué constituye exactamente un trabajo peligroso (OIT, 2018). Por este motivo, en esta investigación se propone la construcción de un índice para calcular el porcentaje de adolescentes que no cuentan con las condiciones mínimas adecuadas para desempeñar un trabajo de acuerdo con las leyes mexicanas, y que, además, permita medir la severidad o peligrosidad de los trabajos desempeñados por los adolescentes. El índice se basará en la metodología de medición multidimensional de Alkire y Foster (2011) utilizando como referencia la LFT y las definiciones de trabajo ligero (para adolescentes de 12 a 14 años), trabajo regular (para jóvenes de 15 a 17 años) y trabajo peligroso (menores de 18 años) por parte de la OIT. En este sentido, proponemos incluir seis dimensiones para crear la medida de trabajo peligroso: 1) horario permitido, 2) problema de salud física debido a su trabajo; 3) el lugar donde labora el adolescente; 4) si el menor requiere cargar cosas pesadas en su trabajo; 5) si el menor ha manifestado problemas de salud mental; y 6) los peligros a los que está expuesto el adolescente en su trabajo. Se estima el índice del trabajo peligroso multidimensional (TPM), que identifica si el adolescente está trabajando y las condiciones laborales que le otorgan.

Los adolescentes analizados en este artículo son aquellos que se encuentran en el mercado laboral y que son hijos del jefe o jefa de hogar. Teniendo esto en cuenta, se estima que el $55.86 \%$ de los adolescentes de 12 a 17 años recibe un salario por el trabajo realizado; en tanto que el $44.14 \%$ no recibe un pago. Del MTI de INEGI (2017b), se obtienen las variables correspondientes al estatus laboral y características del adolescente, mientras que la información de los padres se extrae del cuarto trimestre de la Encuesta Nacional de Ocupación y Empleo (ENOE) del año 2017, INEGI (2017a). Se estima que aproximadamente el $31 \%$ de los trabajadores adolescentes de 12 a 17 años, se encuentra en un TPM, es decir, aproximadamente uno de cada tres adolescentes que se encuentran en el mercado laboral lo hacen en actividades que, debido a su edad, pueden afectarlos al poner en peligro su estado físico y emocional.

Estudiar la influencia de los padres en la ocupación de los hijos es importante, porque trabajar a una edad temprana reduce la probabilidad de que los adolescentes, cuando sean adultos, tengan una mejor ocupación de la que tuvieron sus padres (López-Calva y Macías, 2010). La movilidad intergeneracional de la ocupación depende de los cambios globales en la estructura ocupacional, tal como la pérdida de importancia en la economía por parte del sector agrícola, o por la intensidad neta de la relación de orígenes y destinos ocupacionales (Solís, 2018). En América Latina hay una alta probabilidad de que exista una transmisión de ocupación laboral de padres a hijos (Doruk et al., 2020); específicamente, en México existe un impacto directo de la ocupación del padre en la primera ocupación del hijo (Puga y Solís, 2010).

La pregunta de investigación es estimar si existe una transferencia intergeneracional de ocupaciones de padres a hijos adolescentes. En particular, analizar si influye el sector de actividad en el cual se encuentran trabajando el padre y la madre sobre la situación laboral del adolescente en incorporarse 
en un TPM. La decisión de trabajar y la ocupación elegida por los adolescentes podrían no ser aleatorias, por lo que, se tiene un problema de selección de la muestra. Para corregir este sesgo, se utiliza la metodología de Heckman (1979) aplicando un modelo probit con selección con el fin de obtener estimaciones consistentes y asintóticamente eficientes para todos los parámetros. Los principales resultados indican que, si el menor se encuentra trabajando en el mismo sector económico del padre, la probabilidad de laborar en un TPM es 10.3 puntos porcentuales (pp), más que si lo hace en un sector diferente al padre. En tanto, si el adolescente se encuentra en el mismo sector que la madre, la probabilidad de participar en un TPM se reduce a entre 5.5 y $6.4 \mathrm{pp}$.

Los resultados generales indican que para los adolescentes estar en un trabajo peligroso es redituable, pues la probabilidad de que estén en este tipo de trabajos aumenta con el ingreso que reciben. El ingreso de los padres reduce la probabilidad de que los adolescentes estén en un trabajo peligroso. La influencia del ingreso de la mamá es mayor en los adolescentes de 12 a 14 años más no hay un efecto reductor debido a la escolaridad de la mamá. Por el contrario, para otros grupos analizados, la escolaridad de la mamá es relevante para reducir el trabajo peligroso en las hijas adolescentes y en el grupo de edad de 15 a 17 años, que incluye tanto a hombres como a mujeres. La escolaridad del papá no tiene efecto estadísticamente significativo.

En este contexto, la transmisión intergeneracional de la actividad económica del papá a los hijos e hijas es el principal factor para que los adolescentes estén empleados en un trabajo peligroso, influyendo más en el grupo de adolescentes de menor edad. Por otra parte, la transmisión intergeneracional de la actividad económica de la mamá reduce la probabilidad de que los adolescentes estén en un trabajo peligroso y se encuentra un sesgo de género pues reduce en mayor magnitud la probabilidad en los hijos y no tiene ninguna influencia estadística en las hijas adolescentes. La implicación de política está precisamente en que el contexto familiar es relevante para reducir los riesgos a los que está expuesto el adolescente, como generar una mayor consciencia de los riesgos a los que están expuestos los hijos adolescentes en el lugar de trabajo, cargar cosas pesadas, o bien, la salud física y mental. Por otra parte, en el caso de las hijas adolescentes es posible que el efecto reductor de la mamá en la probabilidad de insertarse en trabajos peligros es no significativo por las labores no remuneradas que las hijas pudieran estar realizando dentro del hogar, y que desafortunadamente no son claramente identificadas con la información disponible.

La contribución del artículo es la de proporcionar un índice que mide el porcentaje de adolescentes que no cuentan con las condiciones mínimas adecuadas para desempeñar un trabajo de acuerdo con las leyes mexicanas, bajo este contexto, el empleo se define como peligroso. Además, el artículo proporciona evidencia de que la ocupación del papá influye en mayor medida para que los adolescentes se inserten en un trabajo peligroso, en comparación con el efecto de la ocupación de la mamá. Entender los factores que inciden en el trabajo peligroso permitiría focalizar los esfuerzos para reducir los riesgos y posiblemente erradicar el trabajo peligroso realizado por los menores de edad.

La estructura del artículo es la siguiente: en la sección 1 se presenta la revisión de literatura. En la sección 2 se construye el índice de trabajo peligroso multidimensional (TPM). En la sección 3 se analizan los datos utilizados sobre el trabajo peligroso. En la sección 4 se explica la metodología. En la sección 5 se muestran los resultados; en la sección 6 están las conclusiones. Posteriormente se muestra la bibliografía. Al final, en el anexo se agregan tres cuadros que complementan el estudio. 


\section{Revisión de literatura}

La movilidad intergeneracional del nivel económico es menor en los países en desarrollo (Solon, 1999). México tiene menores oportunidades de movilidad de bienestar económico en comparación con países industrializados, incluso que Chile y Brasil (Torche, 2010). La baja movilidad social se debe a que los altos niveles de desigualdad son transmitidos de generación en generación (Grajales y Monroy-Gómez-Franco, 2017). La movilidad intergeneracional en México se localiza en el quintil más alto y en el más bajo de la distribución de los recursos, (Torche, 2010).

Diversos estudios indican que México ha presentado movilidad educacional creciente, lo que ha provocado un aumento en las oportunidades de movilidad para toda la población (De Hoyos et al., 2010; Torche, 2010; Solís, 2018). Sin embargo, esta movilidad ascendente se presenta en un entorno en el cual hay cuestionamientos acerca de la calidad de la oferta educativa y en una permanente desigualdad de oportunidades, los más favorecidos han sido quienes tienen mayor escolaridad (Solís, 2018). Por otra parte, existen barreras que dificultan el acceso a educación básica para aquellas personas cuyos padres no tienen educación, aunque estas barreras han disminuido a través del tiempo (Torche, 2010). Algunos autores han encontrado una movilidad educacional positiva, debido a una mayor inversión en la educación pública, que iguala las oportunidades en el largo plazo, y obteniendo una mayor movilidad social en los hogares en donde los jefes de familia tienen baja escolaridad (De Hoyos et al., 2010).

En América Latina hay una transmisión de ocupaciones muy alta del padre al hijo, tanto para hombres como para las mujeres (Doruk et al., 2020). De igual forma, López-Calva y Macías (2010) encuentran que los jóvenes pudieran ingresar al mercado laboral como ayudantes, ya sea en la ocupación del padre o de la madre. La ocupación de la madre es importante en el proceso de movilidad ocupacional de las mujeres, ya que el trabajo de la madre afecta el destino ocupacional de la hija (Rosenfeld, 1978). Solís (2018) indica que la movilidad ocupacional intergeneracional depende de los cambios globales en la estructura ocupacional y en la intensidad neta de la relación entre orígenes y destinos ocupacionales, en donde las mayores diferencias se presentan en los trabajadores agrícolas. Además, las oportunidades de logro ocupacional se encuentran condicionadas por el origen social, los trabajadores que proceden de ocupaciones de mayor categoría tienen mayores posibilidades de permanecer en este tipo de ocupaciones (Solís, 2018). En México, existe un impacto directo de la ocupación del padre en la primera ocupación del hijo, (Zenteno y Solís, 2006; Puga y Solís, 2010). Cortés y Latapí (2005) mencionan que, debido a la rigidez del sistema de movilidad ocupacional mexicano, la ocupación del padre o responsable económico del individuo se vuelve un buen predictor del destino ocupacional.

La mayoría de la literatura acerca del trabajo de los menores de edad se centra en las razones que llevan a los jóvenes a incrustarse en el mercado laboral a una edad temprana, siendo uno de los resultados más importantes que el ingreso de los miembros del hogar determina si los padres o jefes de hogar necesitan enviar a los menores de edad a trabajar para poder subsistir (Basu y Van, 1998). La edad en que los padres iniciaron a trabajar influye en la probabilidad de que los hijos jóvenes se encuentren en el mercado laboral (Emerson y Souza, 2003; Wahba, 2006). Esta probabilidad también es mayor cuando los padres tienen un nivel bajo de escolaridad (Grootaert y Kanbur, 1995; Emerson y Souza, 2003).

Otra gran parte de la literatura se enfoca en las consecuencias de iniciar a trabajar a una edad temprana, siendo los resultados más relevantes los relacionados con la educación y los beneficios 
económicos. Ray (2002) encuentra que una hora más de trabajo remunerado en Ghana está relacionada con un año menos de educación. Heady (2003), utilizando los mismos datos que Ray (2002), encuentra que los trabajadores asalariados obtuvieron resultados más bajos en las pruebas de lectura y matemáticas en comparación con los que no trabajaban. Por su parte, Psacharopoulos (1997) señala que los niños bolivianos que tienen un trabajo asalariado adquieren casi un año menos de educación en comparación con los niños que no se encuentran laborando, en tanto que los niños venezolanos que trabajaban tenían logros educativos de casi dos años menos. Breslin et al. (2007) indican que pueden producirse pérdidas de ingresos entre los trabajadores jóvenes, incluso durante su transición al mercado laboral. En su análisis, Breslin et al. (2007) encuentran que los trabajadores jóvenes que se ausentaron por discapacidad laboral tuvieron menos ganancias en el año posterior a la ausencia en comparación con los jóvenes que no se ausentaron por esta razón. Sin embargo, la literatura también ha encontrado que, en algunos casos, el trabajo a una edad temprana podría generar beneficios en los menores de edad ya que puede originar un desarrollo físico y mental; además, de proporcionar beneficios económicos y sociales para el menor y su familia, siempre y cuando dicho trabajo no afecte la asistencia escolar y el cumplimiento de los deberes escolares (Bourdillon, 2006; Orraca, 2014).

Los adolescentes son más propensos a sufrir lesiones laborales con consecuencias de largo plazo, en comparación con los adultos (Breslin et al., 2003; Steinberg, 2004). La presión que se observa en el trabajo y la exposición al peligro son factores de riesgo que tienen una relación positiva con las lesiones de los adolescentes en el área de trabajo (Evensen et al., 2000). Un análisis sistemático de la literatura evidenció que la fatiga es un factor de riesgo ocupacional en los adolescentes relacionado con accidentes laborales, y puede manifestarse como fatiga física y mental, que ocurre mayormente en los turnos nocturnos (Fischer et al., 2017). Asimismo, se ha encontrado una fuerte relación entre el trabajo juvenil y una deficiente salud mental (Sturrock y Hodes, 2016). Runyan et al. (2007) indican que una gran proporción de adolescentes que se encuentran trabajando realizan tareas que están prohibidas por las leyes federales de trabajo. Del mismo modo, los adolescentes reportan que la capacitación en seguridad, otorgada en el lugar de trabajo, no es eficiente (Zakocs et al., 1998).

En diversos estudios se han mencionado algunos sectores en los cuales los adolescentes sufren más lesiones, entre los cuales se encuentran el sector agrícola, el comercio minorista, sector servicios y la construcción (Hard y Myers, 2006). Dunn et al. (1998) mencionan que los adolescentes se encuentran trabajando principalmente en el comercio minorista y el sector servicios en los cuales están expuestos a equipos y situaciones peligrosas, por lo cual las lesiones físicas son frecuentes. Lipscomb y Li (2001) encuentran que en la industria de la construcción los adolescentes sufren lesiones debido al trabajo en las alturas, el uso de herramientas eléctricas, y vehículos de motor. En el sector agrícola la exposición a pesticidas causa mayor daño a los jóvenes que a la población adulta (Rohlman et al., 2016). Además, se encuentran diferencias por género, los varones son más propensos a peligros en los lugares de trabajo, mientras que las mujeres debido a que manejan mayormente dinero en efectivo tienen mayor riesgo a sufrir un asalto (Dunn et al., 1998; Runyan et al., 2007). Los jóvenes que trabajan en tareas manuales repetitivas simples no requieren largos años de entrenamiento o experiencia, si abandonan la escuela se les dificultaría encontrar, en el futuro, trabajos más calificados, de mayor remuneración y menos peligrosos (Swaminathan, 1998). 


\section{2. ¿Cómo medir el trabajo peligroso?}

Una de las aportaciones de este artículo es la forma en que se construye la variable que mide si el adolescente se encuentra en un trabajo peligroso, para lo cual se utiliza como base la metodología de medición multidimensional de Alkire y Foster (2011). La metodología de Alkire-Foster (AF) es utilizada en México para medir la pobreza multidimensional estimada por el Consejo Nacional de Evaluación de la Política de Desarrollo Social (CONEVAL). Una medida multidimensional AF es un enfoque de conteo en dos pasos; en el primero identifica si una persona está privada o no cumple con el criterio de cada dimensión; y en el segundo, identifica a una persona como pobre de acuerdo con el número de privaciones que experimenta. Alkire y Santos (2013) indican que esta metodología es aplicable a otros fenómenos. En este artículo se aplica la metodología AF para definir y cuantificar el trabajo peligroso multidimensional (TPM). Se considera que un adolescente carece o no cumple con alguna de las dimensiones laborales basadas en los criterios establecidos en la ley federal de trabajo (LFT), y en las definiciones de trabajo ligero (que aplican para menores de 12 a 14 años) y de trabajo regular (que aplican para menores de 15 a 17 años) establecidas por la Organización Internacional del Trabajo $^{3}$. Para definir las dimensiones del índice de TPM se cotejaron los artículos constitucionales de la LFT y las recomendaciones de la OIT con la información disponible del INEGI (2017b). De este proceso se identificaron seis dimensiones que se pueden cuantificar de forma empírica.

Después de definir las dimensiones, se genera una matriz de logro dimensional, $\boldsymbol{X}$, de tamaño $\boldsymbol{n} \boldsymbol{x} \boldsymbol{d}$, donde $\boldsymbol{X}_{\boldsymbol{i}}$ es el logro de la persona $\boldsymbol{i}$ en la dimensión $\boldsymbol{j}$; donde $\boldsymbol{X}_{\boldsymbol{i}}$ es un valor que se encuentra en los números reales positivos. Para cada dimensión j, hay un umbral $\boldsymbol{Z}_{\boldsymbol{j}}$ que se define como el logro mínimo requerido para cumplir con la LFT y las definiciones de la OIT. Tenemos que:

$$
Z=\left(Z_{1}, Z_{2}, Z_{3}, Z_{4}, Z_{5}, Z_{6}\right)
$$

Si $\boldsymbol{X}_{\boldsymbol{i} \boldsymbol{j}}<\boldsymbol{Z}_{\boldsymbol{j}}$ entonces la persona carece o no cumple con esta dimensión; si $\boldsymbol{X}_{\boldsymbol{i} \boldsymbol{j}} \geq \boldsymbol{Z}_{\boldsymbol{j}}$ entonces la persona no carece o sí cumple con las condiciones labores de esta dimensión. Con esta información se obtiene la matriz de privación $\boldsymbol{g}^{\boldsymbol{o}}$, tal que:

$$
\begin{array}{llll}
g_{i j}=1 & \text { si } X_{i j}<Z_{i} & j=1,2, \ldots 6 ; & \mathrm{i}=1,2, \ldots \mathrm{n} \\
g_{i j}=0 & \text { si } X_{i j} \geq \mathrm{Z}_{j} & j=1,2, \ldots 6 ; & \mathrm{i}=1,2, \ldots \mathrm{n}
\end{array}
$$

El siguiente paso es definir el peso de cada dimensión. En este estudio se propone adjudicar el mismo peso a cada dimensión. Entonces, sea w un vector de pesos, tal que:

$$
w=\left(w_{1}, w_{2}, w_{3}, w_{4}, w_{5}, w_{6}\right) \quad \text { donde } w_{j}=1 ; j=1,2, \ldots, 6 .
$$

Después a cada adolescente se le asigna un puntaje de incumplimiento, es decir, se suma el número de dimensiones en las cuales su trabajo no cumple con la LFT o las definiciones de trabajo ligero y trabajo regular de la OIT. El puntaje de incumplimiento viene dado por:

$C_{i}=\sum_{j=1}^{6} W_{j}^{*} g_{i j}^{o}=\sum_{j=1}^{6} g_{i j}^{o}$

Donde $\boldsymbol{C}_{\boldsymbol{i}}$ es un vector de columna de puntajes de incumplimiento para todos los adolescentes:

$$
C=C_{1}, C_{2}, \ldots, C_{n}
$$

3- Definiciones en página 6 del documento metodológico del MTI 2017. Disponible en: https://www.inegi.org.mx/app/ biblioteca/ficha.html?upc $=702825102258$ 
Además de los límites de privación $\boldsymbol{Z}_{\boldsymbol{j}}$, Alkire y Foster (2011) utilizan un segundo umbral, $\boldsymbol{K}$, que denominan corte de pobreza. En este artículo definimos $K$ como el corte de trabajo peligroso. Sea $\rho_{\boldsymbol{k}}$ una función de identificación que depende del vector de logro de cada adolescente $\boldsymbol{X}$, el vector de logro mínimo $\boldsymbol{Z}_{\boldsymbol{j}}$, el vector de peso $\boldsymbol{W}_{\boldsymbol{j}}$ y el umbral de trabajo peligroso $\boldsymbol{K}$. Definimos $\boldsymbol{\rho}_{\boldsymbol{k}}=\mathbf{1}$ si el adolescente se encuentra en un trabajo peligroso y $\boldsymbol{\rho}_{\boldsymbol{k}}=\mathbf{0}$ si el adolescente no labora en un trabajo peligroso, es decir:

$$
\begin{array}{ll}
\rho_{k}\left(X_{i}, Z_{j}, W_{j}, K\right)=1 & \text { si } C_{i} \geq K \\
\rho_{k}\left(X_{i}, Z_{j}, W_{j}, K\right)=0 & \text { si } C_{i}<K
\end{array}
$$

A $\rho_{\boldsymbol{k}}$ se le denomina método de identificación de corte dual o método intermedio. Alkire y Foster (2011) mencionan que dentro del enfoque de conteo existen dos criterios:

a. Criterio de unión. Un joven se encuentra en un trabajo peligroso si incumple al menos una dimensión $\left(\boldsymbol{c}_{\boldsymbol{i}}>\mathbf{0}\right)$.

b. Criterio de intersección. Un adolescente se localiza en un trabajo peligroso si incumple todas las dimensiones $\left(\boldsymbol{c}_{\boldsymbol{i}}>\mathbf{6}\right)$.

Según Alkire y Foster (2011), estos dos criterios pueden ser imprecisos, debido a que el primero clasifica a una proporción muy grande de jóvenes en un trabajo peligroso, en tanto que con el segundo criterio sucede lo contrario, clasifica una proporción muy pequeña de adolescentes en un trabajo peligroso. Alkire y Foster (2011) proponen utilizar un criterio que se encuentre entre estos dos extremos, $\rho_{\boldsymbol{k}}$ incluye ambos métodos como casos especiales. En este artículo definimos que el adolescente está en un trabajo peligroso si se encuentra en un trabajo que incumple con al menos dos dimensiones $(K=2)$, es decir, si la suma de las dimensiones en las que incumple con la LFT y las definiciones de la OIT es igual o mayor a dos $\left(\boldsymbol{C}_{\boldsymbol{i}} \geq \mathbf{2}\right)$. Debido a que se utiliza más de una dimensión para indicar que el adolescente se encuentra en un trabajo peligroso, en este artículo se le denomina índice de trabajo peligroso multidimensional (TPM).

\section{3. $\mathcal{E}$ stimación del trabajo peligroso multidimensional (TPM)}

Para la construcción empírica del TPM se identificaron seis dimensiones que consideran la Ley Federal del Trabajo (LFT), las definiciones de trabajo ligero y trabajo regular definidas por la OIT. Los datos son extraídos del Módulo de Trabajo Infantil (INEGI, 2017b) y se considera a los adolescentes menores de 12 a 17 años que son hijos/as del jefe o jefa de hogar, la mayoría recibe un pago, 55.86\%, y el resto es trabajo no remunerado. El primer paso de conteo consistió en identificar si el trabajo del adolescente incumple con la LFT o las definiciones de la OIT, para lo cual se le asigna el valor de uno, por el contrario, si cumple con las recomendaciones de la dimensión analizada se le asigna el valor de cero. En el Cuadro 1 se indica con detalle la obtención de cada dimensión.

En la primera dimensión se identifica si el adolescente realiza labores nocturnas industriales o si trabaja después de las veintidós horas. Si los adolescentes responden que solo trabajan de día, se les asigna el valor de cero, es decir, cumplen con las condiciones de esta dimensión.

En la segunda dimensión se identifica si el adolescente refiere que tuvo algún problema de salud física debido al trabajo que desempeña, de otra forma se le asigna el valor de cero. 
La tercera dimensión identifica si el adolescente labora en lugares peligrosos o insalubres, que pueden interferir en el desarrollo y la salud tanto física como mental o incluso poner en riesgo la vida del menor. Si el menor trabaja en algún lugar diferente a los mencionados como prohibidos en la LFT se le proporciona el valor de cero.

En la cuarta dimensión se mide si el adolescente cargó algún objeto pesado. Solo para el grupo de adolescentes de menor edad, de 12 a 14 años, se considera que el trabajo del adolescente incumple, sin importar si es trabajo ligero.

En la quinta dimensión se estudia si el menor ha manifestado problemas de salud mental debido a su trabajo. En los adolescentes de 12 a 14 años además se asigna el valor de uno si no puede cumplir con sus labores escolares, aunque si bien es cierto que no acudir a la escuela no necesariamente es un problema de salud mental, se incluye este apartado en el cuestionario de INEGI (2017b). Los adolescentes que refieren no haber sufrido algún problema de salud mental se les asigna un valor de cero.

En la sexta dimensión se incluyen los peligros a los que está expuesto el adolescente en su trabajo. En los adolescentes de 12 a 14 años se asigna el valor de uno a aquellos que manejan vehículos motorizados, realizan posturas forzadas o movimientos repetitivos. Si el adolescente indica no tener peligros en el trabajo se le asigna el valor de cero.

De acuerdo con el Cuadro 1, la dimensión que presenta un mayor porcentaje de jóvenes en trabajos que no cumplen los requerimientos de la LFT y de la OIT es la de peligros a los que está expuesto en el lugar de trabajo, con un $33.62 \%$. En segundo lugar, los adolescentes cargan cosas pesadas, levanta objetos o materiales pesados en su trabajo, $25.27 \%$. En tercer lugar, el $21.16 \%$ de los jóvenes trabajadores tuvieron algún problema de salud física, en tanto que el $16.42 \%$ ha presentado problemas de salud mental. Aunque en poca proporción, pero no por eso menos importante, el $6.27 \%$ trabaja en un horario no adecuado para su edad, así como el 5.46\% lo hace en un lugar no recomendable para un adolescente.

La metodología multidimensional de Alkire y Foster (2011) implica dos criterios de decisión para estimar las condiciones laborales que no se cumplen simultáneamente. El primero es la ponderación asignada a cada dimensión del índice y la segunda es el umbral de condiciones laborales mínimas permitidas. Como primer ejercicio se asume que las dimensiones que componen el índice tienen la misma ponderación. Como segundo ejercicio se presentan los distintos umbrales de cumplimiento mínimo de condiciones laborales para estimar el porcentaje de adolescentes en trabajo peligroso, como se muestra en el Cuadro 2.

En el Cuadro 2 se presenta el porcentaje de adolescentes en trabajo peligroso multidimensional (TPM) utilizando diferentes criterios para seleccionar dimensiones. Si definimos al TPM, como aquel en donde no se cumple al menos una dimensión, $K=\mathbf{1}$, tendríamos un alto porcentaje de adolescentes en un TPM (58.9\%), es por eso por lo que Alkire y Foster (2011) no recomiendan utilizar este criterio ya que en este caso sobrestimaría el porcentaje de adolescentes en un trabajo peligroso. Usando la medida de que el trabajo del joven incumple en al menos dos dimensiones, $K=\mathbf{2}$, se estima que el $30.53 \%$ se encuentra en un TPM. Si se utiliza un corte de trabajo peligroso igual a tres, $\boldsymbol{K}=\mathbf{3}$, entonces se obtiene un porcentaje de adolescentes en TPM de $13.87 \%$. 


\section{Cuadro 1}

\section{Dimensiones que componen el índice de trabajo peligroso}

\begin{tabular}{|c|c|c|}
\hline DIMENSIÓN & LEY FEDERAL DEL TRABAJO (LFT) & CRITERIO DE INCUMPLIMIENTO \\
\hline $\begin{array}{l}\text { Horario laboral } \\
(6.27 \%)\end{array}$ & $\begin{array}{l}\text { Artículo } 176 \text { de la LFT indica que queda } \\
\text { prohibido que el menor realice labores } \\
\text { nocturnas industriales o trabaje después de } \\
\text { las veintidós horas. }\end{array}$ & $\begin{array}{l}\text { Si el menor responde que trabaja de noche, rola } \\
\text { turnos o su horario es mixto. }\end{array}$ \\
\hline $\begin{array}{l}\text { Problema de } \\
\text { salud física* } \\
(21.16 \%)\end{array}$ & $\begin{array}{l}\text { Según el Artículo 175, queda prohibida la } \\
\text { utilización del trabajo de los menores de } \\
\text { edad en labores peligrosas o insalubres que } \\
\text { pongan en riesgo su salud física y mental de } \\
\text { los menores. }\end{array}$ & $\begin{array}{l}\text { Si el adolescente responde que ha tenido algún } \\
\text { problema de salud por culpa de su trabajo. }\end{array}$ \\
\hline $\begin{array}{l}\text { Lugar de trabajo } \\
(5.46)\end{array}$ & Artículos 175 y 176 de la LFT. & $\begin{array}{l}\text { Si el lugar de trabajo del adolescente es un bar, } \\
\text { cantina, centro nocturno, centro de masajes, } \\
\text { mina, río, lago, mar, montaña, sobre andamios o } \\
\text { pisos elevados, un espacio sin ventilación o poco } \\
\text { iluminado, vía pública o un depósito de basura. }\end{array}$ \\
\hline $\begin{array}{l}\text { Carga cosas } \\
\text { pesadas* } \\
(25.27 \%)\end{array}$ & $\begin{array}{l}\text { En el Artículo } 176 \text { de la LFT, se consideran } \\
\text { las labores productivas prohibidas en las } \\
\text { industrias gasera, del cemento, minera, } \\
\text { del hierro y el acero, petrolera, nuclear, } \\
\text { ladrillera, vidriera, entre otras. }\end{array}$ & $\begin{array}{l}\text { En el rango de } 12 \text { a } 14 \text { años se considera que } \\
\text { incumple si mencionó que cargó algo pesado en el } \\
\text { trabajo. } \\
\text { Para el rango de } 15 \text { a } 17 \text { años, se basa en el Artículo } \\
176 \text { de la LFT. }\end{array}$ \\
\hline $\begin{array}{l}\text { Problemas de } \\
\text { salud mental* } \\
(16.42 \%)\end{array}$ & $\begin{array}{l}\text { En el Artículo } 175 \text { de la LFT indica que } \\
\text { queda prohibida la utilización del trabajo } \\
\text { de los menores de edad en trabajos que } \\
\text { son capaces de actuar sobre la vida, el } \\
\text { desarrollo y la salud física y mental. }\end{array}$ & $\begin{array}{l}\text { Si los adolescentes han sufrido estrés, han sufrido } \\
\text { maltrato, amenazas e insultos, su trabajo les } \\
\text { provoca angustia o miedo de que algo les pase, } \\
\text { así como también cansancio o agotamiento, por } \\
\text { motivos de trabajo. } \\
\text { En los adolescentes de } 12 \text { a } 14 \text { años además se } \\
\text { asigna el valor de uno si no puede cumplir con sus } \\
\text { labores escolares. }\end{array}$ \\
\hline $\begin{array}{l}\text { Peligros en lugar } \\
\text { de trabajo* } \\
(33.62 \%)\end{array}$ & $\begin{array}{l}\text { Artículos } 175 \text { y } 176 \text { de la LFT; así como las } \\
\text { definiciones de la OIT. }\end{array}$ & $\begin{array}{l}\text { Si los adolescentes están expuestos a fuego, } \\
\text { explosivos, ruido excesivo, vibraciones, } \\
\text { humedad, temperatura extrema, herramientas } \\
\text { punzo-cortantes, productos químicos, rayos } \\
\text { X, ultravioleta, animales peligrosos, plantas } \\
\text { venenosas, residuos o desechos peligrosos. } \\
\text { En los adolescentes de } 12 \text { a } 14 \text { años también se } \\
\text { asigna el valor de uno a aquellos que manejan } \\
\text { vehículos motorizados, realizan posturas forzadas o } \\
\text { movimientos repetitivos. }\end{array}$ \\
\hline
\end{tabular}

Fuente: Elaboración propia con base en LFT (Secretaría del Trabajo y Previsión Social, 2019). Nota: La incidencia de cada dimensión se muestra entre paréntesis, no suman 100 porque el empleo adolescente puede no cumplir con más de una condición laboral. * Para los adolescentes de 12 a 14 años no cumple con la definición de trabajo ligero como indica la OIT (2018). 
Cuadro 2

Porcentaje de adolescentes en TPM según distintos criterios de dimensiones

\begin{tabular}{|c|c|c|c|c|c|}
\hline & $K=1$ & $K=2$ & $K=3$ & $K=4$ & $K=5$ \\
\hline \multirow{2}{*}{ General } & $58.90 \%$ & $30.53 \%$ & $13.87 \%$ & $4.50 \%$ & $0.38 \%$ \\
\hline & {$[1,030,165]$} & {$[533,999]$} & {$[242,633]$} & {$[78,695]$} & {$[6,634]$} \\
\hline \multirow{2}{*}{ Hombres } & $67.76 \%$ & $36.85 \%$ & $16.97 \%$ & $5.78 \%$ & $0.49 \%$ \\
\hline & {$[846,131]$} & {$[460,081]$} & {$[211,940]$} & {$[72,135]$} & {$[6,175]$} \\
\hline \multirow{2}{*}{ Mujeres } & $36.79 \%$ & $14.78 \%$ & $6.14 \%$ & $1.31 \%$ & $0.09 \%$ \\
\hline & {$[184,034]$} & {$[73,918]$} & {$[30,693]$} & {$[6,560]$} & [459] \\
\hline \multirow{2}{*}{12 a 14 años } & $60.25 \%$ & $29.58 \%$ & $13.27 \%$ & $5.95 \%$ & $0.32 \%$ \\
\hline & {$[281,476]$} & {$[138,188]$} & {$[61,999]$} & {$[27,814]$} & {$[1,472]$} \\
\hline \multirow{2}{*}{15 a 17 años } & $58.41 \%$ & $30.88 \%$ & $14.09 \%$ & $3.97 \%$ & $0.40 \%$ \\
\hline & {$[748,689]$} & {$[395,811]$} & {$[180,634]$} & {$[50,881]$} & {$[5,162]$} \\
\hline
\end{tabular}

Fuente: Elaboración propia con datos del MTI, INEGI (2017b).

Esta última cifra es similar a la estimada en INEGI (2018) para los adolescentes de 15 a 17 años, pero es condicional a que el mínimo de prohibiciones en la LFT sea al menos tres, un criterio que subestima la situación laboral de adolescentes. En el Cuadro 2, se observa que conforme aumenta el umbral o corte de trabajo peligroso, $\boldsymbol{K}$, disminuye el porcentaje de adolescentes en un TPM. En este estudio se asume que, si el trabajo del adolescente no cumple con al menos dos de las seis dimensiones descritas anteriormente, entonces, el adolescente se encuentra en un TPM. No existe un consenso sobre cuál es el corte más adecuado a elegir. Un criterio conservador es elegir el valor con el menor cambio porcentual de TPM entre dos cortes consecutivos. Esto es, cuando se consideran dos dimensiones $(\mathrm{k}=2)$ en lugar de una dimensión $(\mathrm{k}=1)$, la reducción estimada de TPM es de $-48 \%$, en tanto, el cambio en valor absoluto aumenta progresivamente al incluir una dimensión con relación a la inmediata anterior; cuando se consideran de tres a dos dimensiones, la reducción estimada es de $-55 \%$, de cuatro a tres dimensiones la reducción en TPM es de $-68 \%$, y de cinco a cuatro dimensiones, la reducción es de $-92 \%$. Utilizando este criterio encontramos que el $30.53 \%$ de los adolescentes se encuentran en un TPM.

En el Cuadro 2 también se presentan porcentajes de adolescentes en un TPM al separar por género y grupos de edad, en diferentes criterios para la selección. Asumiendo el corte de dos dimensiones, se encuentran porcentajes menores a los estimados con solo una dimensión, y se mantiene el criterio del cambio porcentual menor entre cortes por grupos de edad, solo con excepción de las mujeres adolescentes. Se encuentra una gran diferencia entre hombres y mujeres, pues los hombres trabajaban mayormente en TPM en comparación con las mujeres, bajo cualquier criterio de selección. El porcentaje de adolescentes hombres que incumplen dos dimensiones es de $36.85 \%$, en tanto que para las mujeres dicho porcentaje es de $14.78 \%$. Al dividir a los jóvenes por grupos de edad, las proporciones de adolescentes en TPM son similares, en el grupo de 12 a 14 años se estima en $29.58 \%$ y en el grupo de 15 a 17 años es de $30.88 \%$.

En el cuadro A1 del anexo se presentan tres escenarios adicionales cambiando las ponderaciones en cada dimensión y manteniendo el mismo criterio de incumplimiento de al menos dos condiciones laborales. No existe un consenso sobre cuál de las dimensiones debe tener un mayor peso, pues esta 
asignación implica reducir la ponderación de otras de las dimensiones. En el primer ejercicio se asumió la misma ponderación, como segundo ejercicio se le asignó el doble del valor a las dimensiones que se basan en dos artículos de la LFT (artículos 175 y 176), como el lugar y el estar expuesto a los peligros del trabajo. Por otra parte, la salud física y la salud mental son las dimensiones más correlacionadas (0.432), por lo que, el tercer ejercicio se asignó una menor ponderación a estas dos dimensiones, y como cuarto ejercicio se les dio una mayor ponderación (Sturrock y Hodes, 2016).

La distribución geográfica de la incidencia del TPM, se muestra en el Mapa 1. Se distingue una heterogeneidad entre las entidades federativas mexicanas, pues existe una diferencia de aproximadamente 47 puntos porcentuales entre las entidades con menor y mayor porcentaje de adolescentes en un TPM. El promedio nacional de adolescentes en un TPM es de (31.1\%). En cinco estados existe una alta incidencia de TPM, de al menos 40\%, en diez estados entre el 30 y $40 \%$ de los adolescentes se encuentran en TPM, en nueve estados está entre 20 y $30 \%$, y en ocho estados se estiman los porcentajes más bajos, entre 14 y $20 \%$.

Chiapas es la entidad con mayor porcentaje de adolescentes en un TPM con 61\%, casi el doble del promedio nacional. Campeche, es el estado con el segundo mayor porcentaje de adolescentes con TPM, con 51\%. En estas dos entidades más de la mitad de la fuerza laboral adolescente labora en actividades que no cumplen al menos dos de las seis dimensiones laborales. Michoacán, Nayarit y Zacatecas también muestran alta incidencia de TPM con aproximadamente el $45 \%$ y $49 \%$, cifras cercanas a la mitad de la población.

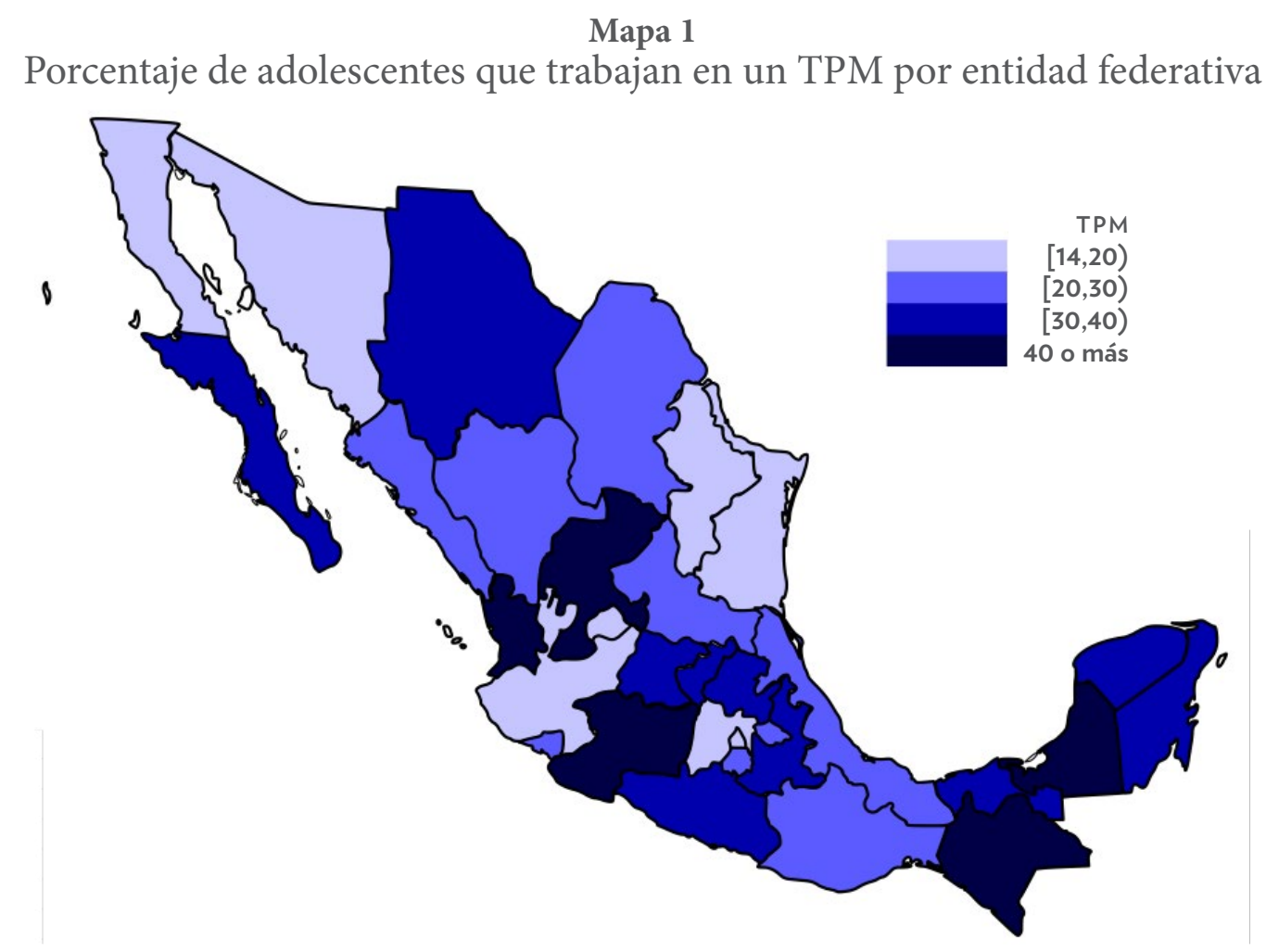

Fuente: Elaboración propia con datos del MTI, INEGI (2017b). 
Jalisco y Nuevo León, son las entidades con menor porcentaje de jóvenes en TPM con 14\% y 15\% respectivamente. Aguascalientes, Ciudad de México, Baja California y el Estado de México también cuentan con baja incidencia de TPM, en promedio 17\%. También Tamaulipas y Sonora son entidades con un porcentaje relativamente bajo de menores en un TPM, ya que cuentan con $18 \%$ y $19.9 \%$ de la fuerza laboral juvenil en esta condición.

Con el fin de responder la pregunta de investigación, es importante analizar el porcentaje de adolescentes que se encuentran trabajando en el mismo sector de actividad económica que sus padres. En el Cuadro 3 se presenta esta información a nivel nacional. Las estadísticas indican que 0.73 millones de jóvenes trabajan en el mismo sector que el padre, en tanto que aproximadamente 0.51 millones de adolescentes laboran en el mismo sector que la mamá. Si analizamos las proporciones de jóvenes en un TPM, notamos que, 39.14\% de los adolescentes, que reportaron trabajar en el mismo sector que el papá, se encuentran en un TPM. En menor proporción, 22.65\%, se estima el porcentaje de adolescentes que laboran en el mismo sector que la madre y se encuentran en un TPM.

Al separar por género, se encuentra que aproximadamente 0.6 millones de adolescentes varones se encuentran laborando en el mismo sector de la actividad económica utilizando la clasificación de sector-SCIAN ${ }^{4}$ que el papá, de los cuales el $43.46 \%$ lo hace en un TPM. En tanto, alrededor de 0.28 millones lo hace en el mismo sector que la madre, siendo el 27.61\% los que laboran en un TPM. En el caso de las mujeres, aproximadamente 0.12 millones laboran en el mismo sector-SCIAN que el padre, de los cuales el 19\% lo hace en un TPM; y 0.23 millones en el mismo sector que la mamá, siendo de $16.58 \%$, una menor incidencia de TPM comparado con el porcentaje obtenido si la hija se encuentra en el mismo sector que el padre. En lo que respecta a la edad, el $37.70 \%$ de los adolescentes de 12 a 14 años, que se encuentran laborando en el mismo sector-SCIAN que el padre, están empleados en un TPM; en tanto que el $20.75 \%$ está en un TPM y trabaja en el mismo sector que la madre. La incidencia de TPM es ligeramente mayor en el grupo de 15 a 17 años, ya que el 39.79\% está en un TPM, dado que se encuentra en el mismo sector-SCIAN que el papá, en tanto que el $23.67 \%$ se encuentra en este tipo de trabajos, condicional a que labora en el mismo sector que la mamá.

Hay que tener en cuenta que no todos los sectores clasificados como TPM para los adolescentes, son considerados peligrosos para los adultos y los adolescentes conjuntamente. Para clasificar a cada sector de actividad se utiliza una variable para medir la tasa de riesgo de los sectores para clasificarlos en riesgo bajo o nulo, medio o alto. Primero se obtiene el riesgo de trabajo para cada sector sumando los accidentes, lesiones y enfermedades que han tenido los adolescentes a causa del trabajo que realizan. Posteriormente se divide esta suma entre el total de adolescentes trabajando en cada sector y se le denomina tasa de riesgo del sector. En el Cuadro 4 se muestra la tasa de riesgo, el número de adolescentes que se encuentran trabajando y la incidencia de TPM para cada sector de actividad económica. En cinco actividades económicas se concentra el $85.18 \%$ de los adolescentes que trabajan. Una tercera parte de los adolescentes que trabajan se encuentran en la agricultura, ganadería y pesca; en el comercio al por menor se encuentra el 20.8\%, en la industria manufactura está empleado el 12.94\%, en los servicios de hospedaje y de preparación de alimentos y bebidas se encuentra el 11.55\%, y en el sector de la construcción está el 6.8\%. Solo la agricultura y la construcción muestran porcentajes altos de adolescentes en TPM, de $47.75 \%$ y $61.19 \%$, respectivamente, y además tienen altas tasa de riesgo laboral. 
Cuadro 3

Porcentaje de adolescentes laborando en el mismo sector-SCIAN que padre/madre

\begin{tabular}{|l|l|l|}
\hline \multirow{5}{*}{ General } & $\begin{array}{l}\text { MISMO SECTOR- } \\
\text { SCIAN PAPA }\end{array}$ & $\begin{array}{l}\text { MISMO SECTOR- } \\
\text { SCIAN MAMÁ }\end{array}$ \\
\cline { 2 - 3 } & TPM & TPM \\
\hline \multirow{3}{*}{ Hombres } & $39.14 \%$ & $22.65 \%$ \\
\cline { 2 - 3 } & {$[287,813]$} & {$[116,826]$} \\
\hline \multirow{3}{*}{ Mujeres } & $43.46 \%$ & $27.61 \%$ \\
\cline { 2 - 3 } & {$[263,153]$} & {$[78,319]$} \\
\hline \multirow{3}{*}{12 a 14 años } & $19.00 \%$ & $16.58 \%$ \\
\cline { 2 - 3 } & {$[24,660]$} & {$[38,507]$} \\
\hline \multirow{2}{*}{15 a 17 años } & $37.70 \%$ & $20.75 \%$ \\
\cline { 2 - 3 } & {$[86,624]$} & {$[37,566]$} \\
\cline { 2 - 3 } & $39.79 \%$ & $23.67 \%$ \\
\hline
\end{tabular}

Fuente: Elaboración propia con datos del MTI, INEGI (2017b).

Analizando las estadísticas del Cuadro 4 se puede ver que el sector con la mayor tasa de riesgo laboral es la minería, $18.8 \%$, aunque un porcentaje menor al 1\% de los adolescentes se emplea en este sector. El sector de actividad del comercio al por mayor es de riesgo laboral alto y el porcentaje de adolescentes en situación de TPM es de 32.55\%, pero está empleado solo el 1.5\% de los adolescentes que trabajan. Finalmente, hay sectores en donde el riesgo laboral es medio y que tienen porcentajes de entre 20 y $31 \%$ de adolescentes en TPM, en servicios de esparcimiento, culturales y deportivos (30.97\%), en la manufactura $(24.87 \%)$ y en servicios de hospedaje y de preparación de alimentos y bebidas. La variabilidad que se encuentra entre el porcentaje de adolescentes en TPM y el porcentaje que comparten la misma actividad económica con sus padres, así como las variables socioeconómicas permitirán entender la transferencia intergeneracional de la ocupación de los padres a sus hijos adolescentes.

\section{Cuadro 4}

Tasa de riesgo, adolescentes trabajando e incidencia de TPM por SCIAN

\begin{tabular}{|c|c|c|c|c|}
\hline SECTOR-SCIAN & $\begin{array}{l}\text { TASA DE } \\
\text { RIESGO }\end{array}$ & $\begin{array}{l}\text { RIESGO DEL } \\
\text { SECTOR }\end{array}$ & $\begin{array}{l}\text { ADOLESCENTES } \\
\text { TRABAJANDO } \\
\text { EN ESTE SECTOR }\end{array}$ & $\begin{array}{l}\text { ADOLESCENTES } \\
\text { EN TPM }(\%)\end{array}$ \\
\hline 1.- Agricultura, ganadería... & 6.6 & Alto & 569,265 & $47.75 \%$ \\
\hline 2.-Minería & 18.8 & Alto & 255 & $18.82 \%$ \\
\hline $\begin{array}{l}\text { 3.- Generación y distribución de } \\
\text { electricidad, suministro de agua y gas }\end{array}$ & 0.0 & Bajo o nulo & 592 & $0.00 \%$ \\
\hline 4.- Construcción & 5.7 & Alto & 116,940 & $61.19 \%$ \\
\hline 5.- Industrias manufactureras & 4.1 & Medio & 222,621 & $24.87 \%$ \\
\hline 6.-Comercio al por mayor & 4.2 & Alto & 25,886 & $32.55 \%$ \\
\hline 7.-Comercio al por menor & 2.1 & Medio & 357,863 & $11.45 \%$ \\
\hline 8.-Transportes, correos y almacenamiento & 4.0 & Medio & 21,905 & $18.34 \%$ \\
\hline 9.-Información en medios masivos & 0.0 & Bajo o nulo & 2,252 & $12.83 \%$ \\
\hline 10.-Servicios financieros y de seguros & 0.0 & Bajo o nulo & 588 & $0.00 \%$ \\
\hline
\end{tabular}




\begin{tabular}{|l|l|l|l|l|}
\hline SECTOR-SCIAN & $\begin{array}{l}\text { TASA DE } \\
\text { RIESGO }\end{array}$ & $\begin{array}{l}\text { RIESGO DEL } \\
\text { SECTOR }\end{array}$ & $\begin{array}{l}\text { ADOLESCNTES } \\
\text { TRABA JANDO } \\
\text { EN ESTE SECTOR }\end{array}$ & $\begin{array}{l}\text { ADOLES TPM (\%) } \\
\text { ENES }\end{array}$ \\
\hline $\begin{array}{l}\text { 11.-Servicios inmobiliarios y de alquiler de } \\
\text { bienes }\end{array}$ & 5.0 & Alto & 8,549 & $16.12 \%$ \\
\hline $\begin{array}{l}\text { 12.-Servicios profesionales, científicos y } \\
\text { técnicos }\end{array}$ & 0.0 & Bajo o nulo & 4,850 & $11.55 \%$ \\
\hline $\begin{array}{l}\text { 14.-Servicios de apoyo a los negocios y } \\
\text { manejo de desechos }\end{array}$ & 2.9 & Medio & 24,847 & $18.27 \%$ \\
\hline $\begin{array}{l}\text { 15.-Servicios educativos } \\
\text { 16.-Servicios de salud y de asistencia social }\end{array}$ & 0.0 & Bajo o nulo & 13,029 & $9.39 \%$ \\
\hline $\begin{array}{l}\text { 17.-Servicios de esparcimiento, culturales y } \\
\text { deportivos }\end{array}$ & 3.3 & Bajo o nulo & 6,528 & $19.53 \%$ \\
\hline $\begin{array}{l}\text { 18.-Servicios de hospedaje y de } \\
\text { preparación de alimentos y bebidas }\end{array}$ & 3.8 & Medio & 24,824 & $30.97 \%$ \\
\hline $\begin{array}{l}\text { 19.- Otros servicios, excepto actividades } \\
\text { gubernamentales }\end{array}$ & 4.5 & Alto & 198,631 & $20.40 \%$ \\
\hline $\begin{array}{l}\text { 20.- Actividades gubernamentales y de } \\
\text { organismos internacionales }\end{array}$ & 0.0 & Bajo o nulo & 4,809 & $15.57 \%$ \\
\hline
\end{tabular}

Fuente: Elaboración propia con datos del MTI, INEGI (2017b). Nota: En la clasificación 13 de corporativos no se encuentran menores trabajando.

\section{Metodología}

Este artículo examina la influencia del sector de actividad económica de los padres sobre sus hijos adolescentes que trabajan. La intuición indica que el sector de actividad económica en el que se encuentran los padres influye en el sector en el que se ubicará el adolescente, al menos en su primer empleo. Los adolescentes podrían entrar al mercado laboral influidos por la actividad económica realizada por los padres, porque los acompañan o van de ayudantes. Sin embargo, si alguno de los padres se encuentra en un sector donde realizan actividades peligrosas o riesgosas, o bien, aun y cuando la actividad no sea riesgosa para los padres sí podría poner en riesgo a los trabajadores adolescentes.

En este artículo se estima la probabilidad de que el adolescente se encuentre en un TPM. Es posible tener un problema de sesgo de selección de la muestra, pues los trabajadores analizados podrían estar autoseleccionados. Es decir, los adolescentes que se encuentran en el mercado laboral no ingresan de manera aleatoria, sino que existen circunstancias que los llevan a esta situación. Heckman (1979) analiza el sesgo de selección de muestra como un error de especificación y presenta un método de estimación simple y consistente que elimina dicho error para el caso de muestras censuradas. Basados en Heckman (1979), Van de Ven y Van Pragg (1981) proporcionan una introducción y una explicación del modelo probit con selección, indicando que dichos modelos proporcionan estimaciones consistentes y asintóticamente eficientes para todos los parámetros. Para estimar nuestro modelo necesitamos dos ecuaciones: la ecuación probit (observada) y la ecuación de selección. Para que el modelo esté identificado, la ecuación de selección debe tener al menos una variable que no esté en la ecuación probit, variable de exclusión; de lo contrario, el modelo se identifica solo por su forma funcional y los coeficientes no tienen interpretación estructural (Van de Ven y Van Pragg, 1981). 
La ecuación probit que se desea estimar indica la relación que existe entre el tipo de trabajo que tiene el adolescente y si dicho trabajo se realiza en el mismo sector de actividad económica en el que trabaja alguno de sus padres, variable para medir la transferencia intergeneracional; así también se incluyen otras variables relevantes para medir la probabilidad de que el menor trabaje en un TPM. La ecuación que se estima es la siguiente:

$T P M_{i}=\beta_{0}+\beta_{1} D M S e c_{p \alpha p a ́ i}+\beta_{2} D M S e c_{m \alpha m a ́ i}+\beta_{3} X_{i}+\beta_{4} W_{i}+\varepsilon_{i}$

Donde $T P M_{i}=1$ si el adolescente se encuentra en un trabajo peligroso multidimensional o $T P M_{i}$ $=0$ si se encuentra en un trabajo no peligroso multidimensional. $D M S e c_{p \alpha p a ́}=1$ si el adolescente se encuentra trabajando en el mismo sector que el papá, 0 de otra forma; $D M S e c_{m \alpha m a ́ i}=1$ si el joven trabaja en el mismo sector que la mamá, 0 si se encuentra en un sector diferente; $\boldsymbol{X}_{\boldsymbol{i}}$ es un vector de características del adolescente tales como género, ingreso mensual, edad a la que empezó a trabajar, así como también si cuenta con seguro médico por parte del trabajo; $\boldsymbol{W}_{\boldsymbol{i}}$ es un vector que incluye características de los padres: escolaridad e ingreso. Se agregan variables dicotómicas por región y $\varepsilon_{i}$ es el término de error.

En la ecuación de selección, la variable dependiente es la que indica si el adolescente trabaja (igual a uno) o si no trabaja (igual a cero); se añaden la edad del menor, edadi, y si el adolescente se encuentra estudiando, d_estudia $i$, para que el modelo esté bien identificado. La ecuación de selección es la siguiente:

Trabaja $_{i}=\gamma_{0}+\gamma_{1}$ edad $i+\gamma_{2} d_{-}$estudia $i+\gamma_{3} X_{i}+\gamma_{4} W_{i}+u_{i}$

Hay que señalar que en la ecuación de selección no se incluyen aquellas variables que se tienen solo para los adolescentes que se encuentran trabajando, tales como, si el adolescente se encuentra trabajando en el mismo sector que el papá o mismo sector que la mamá, su ingreso mensual, la edad a la que empezó a trabajar y si cuenta con seguro médico por parte del trabajo.

\section{Resultados}

La estrategia empírica busca la identificación de la transmisión intergeneracional de la actividad económica entre padres e hijos y cómo esta ocupación influye en el tipo de trabajo que desempeña el adolescente. Cabe recordar que la atención se centra en los trabajos peligrosos multidimensionales, ya que, si el adolescente se encuentra trabajando en la misma ocupación que alguno de los padres, y si estos se encuentran en un trabajo riesgoso o peligroso, el joven también se encontrará en este tipo de labores, aunque también pudiera pasar que el trabajo no es peligroso para el padre o madre, pero sí lo es para el adolescente debido a su corta edad. Los resultados de la ecuación de selección se muestran en el anexo.

En el Cuadro 5 se presentan los resultados de los efectos marginales de las variables explicativas en la probabilidad de que el adolescente se encuentre en un TPM. Los resultados indican que los hombres tienen una probabilidad mayor, en comparación con las mujeres, de pertenecer a un TPM, la interpretación se hará en puntos porcentuales (pp). Específicamente, la probabilidad de trabajar en un TPM es aproximadamente $12.6 \mathrm{pp}$ mayor en los varones. Tomando en cuenta todos los modelos estimados, los adolescentes hombres tienen una mayor probabilidad, de entre 12.6 pp y 13.8 pp, en comparación con las mujeres adolescentes de laborar en un TPM. Además, existe una relación positiva entre el ingreso 
mensual del adolescente y laborar en un TPM. Si el ingreso de los adolescentes se incrementa en mil pesos, la probabilidad de estar en un TPM se incrementa en $3.3 \mathrm{pp}$. Analizando los coeficientes estimados para esta variable en los cuatro modelos, podemos indicar que, si hay un aumento de mil pesos en el ingreso de los adolescentes, manteniendo todo lo demás constante, la probabilidad de laborar en un TPM aumentaría, en promedio, entre 2.6 pp y 3.4 pp. La edad a la que empezó a trabajar el adolescente también tiene un efecto significativo en la probabilidad de encontrarse en un TPM, estas dos variables tienen una relación inversa. Si la edad en la que el adolescente empezó a trabajar se incrementa en un año más, la probabilidad de encontrarse en un TPM se reduce, en promedio, entre 2.3 pp y 3.2 pp. Como variable proxy de formalidad en el empleo se utiliza una variable indicativa si el adolescente cuenta con seguro médico otorgado por el empleador. Se encuentra que, si el adolescente tiene seguro médico, la probabilidad de estar en un TPM se reduce entre 14.9 pp y 16.4 pp, ceteris paribus.

\section{Cuadro 5}

\section{Efectos marginales del TPM}

\begin{tabular}{|c|c|c|c|c|}
\hline & MODELO A & MODELO B & MODELO C & MODELO D \\
\hline \multirow{2}{*}{ Género } & $0.131^{* * *}$ & $0.138^{* * *}$ & $0.138^{* * *}$ & $0.126^{* * *}$ \\
\hline & {$[4.48]$} & {$[4.58]$} & {$[4.61]$} & [4.17] \\
\hline \multirow{2}{*}{ Ingreso mensual del adolescente } & $0.0000264^{* * *}$ & $0.0000345^{* * *}$ & $0.0000274^{* * *}$ & $0.0000333^{* * *}$ \\
\hline & [4.31] & {$[5.54]$} & [4.37] & [5.30] \\
\hline \multirow{2}{*}{ Edad a la que empezó a trabajar } & $-0.0321^{* * *}$ & $-0.0237^{* * *}$ & $-0.0297^{* * *}$ & $-0.0235^{* * *}$ \\
\hline & {$[-7.02]$} & {$[-4.65]$} & {$[-6.08]$} & {$[-4.56]$} \\
\hline \multirow{2}{*}{ Dummy de seguro médico } & $-0.155^{* *}$ & $-0.164^{* *}$ & $-0.149^{* *}$ & $-0.158^{* *}$ \\
\hline & {$[-2.34]$} & {$[-2.37]$} & {$[-2.17]$} & {$[-2.27]$} \\
\hline \multirow{2}{*}{$\begin{array}{l}\text { Dummy si adolescente trabaja en el } \\
\text { mismo sector que papá }\end{array}$} & & $0.103^{* * *}$ & & $0.103^{* * *}$ \\
\hline & & [4.62] & & {$[4.61]$} \\
\hline \multirow{2}{*}{ Ingreso papá } & & $-0.00000438^{*}$ & & $-0.00000381^{*}$ \\
\hline & & {$[-1.93]$} & & {$[-1.68]$} \\
\hline \multirow{2}{*}{ Escolaridad papá } & & $-0.00681^{* *}$ & & -0.00261 \\
\hline & & {$[-2.06]$} & & {$[-0.74]$} \\
\hline \multirow{2}{*}{$\begin{array}{l}\text { Dummy si adolescente trabaja en el } \\
\text { mismo sector que mamá }\end{array}$} & & & $-0.0554^{* *}$ & $-0.0639^{* *}$ \\
\hline & & & {$[-2.07]$} & {$[-2.40]$} \\
\hline \multirow{2}{*}{ Ingreso mamá } & & & $-0.0000100^{* * *}$ & $-0.00000774^{\star *}$ \\
\hline & & & {$[-2.75]$} & {$[-2.29]$} \\
\hline \multirow{2}{*}{ Escolaridad mamá } & & & $-0.00996^{* * *}$ & $-0.00788^{* *}$ \\
\hline & & & {$[-2.79]$} & {$[-2.11]$} \\
\hline Dummies por región & $\mathrm{Si}$ & $\mathrm{Si}$ & $\mathrm{Si}$ & $\mathrm{Si}$ \\
\hline Constante & $\mathrm{Si}$ & $\mathrm{Si}$ & $\mathrm{Si}$ & $\mathrm{Si}$ \\
\hline Obs. & 28,301 & 28,301 & 28,301 & 28,301 \\
\hline Seleccionadas & 3,890 & 3,890 & 3,890 & 3,890 \\
\hline No selecc. & 24,411 & 24,411 & 24,411 & 24,411 \\
\hline Wald chi2 & 96.36 & 111.35 & 110.31 & 126.28 \\
\hline
\end{tabular}

Fuente: Elaboración propia con datos del MTI, INEGI (2017b) y Cuarto trimestre ENOE, INEGI (2017a). Nota: Significancia estadística ${ }^{\star} 90 \%,{ }^{* *} 95 \%,{ }^{* *} 99 \%$. 
Cuando el adolescente trabaja en la misma actividad económica del padre, la probabilidad de que esté empleado en un TPM aumenta en 10.3 pp, manteniendo todo lo demás constante. Si el ingreso mensual del padre aumenta en mil pesos, reduce la probabilidad de que su hijo labore en un TPM de entre 0.38 pp y 0.43 pp. Se encuentra un efecto similar para la escolaridad del padre, ya que en el modelo en el cual solo se añaden las variables del padre resulta significativa y con signo negativo. Por otro lado, las variables de la madre resultan significativas y muestran una relación inversa con la variable dependiente. Tanto un aumento en el ingreso y la escolaridad de la madre están asociadas a una disminución de la probabilidad de que su hijo se encuentre en un TPM. Pero, lo más relevante es que si el joven se encuentra en el mismo sector que la madre, la probabilidad de laborar en un TPM se reduciría entre 5.5 pp y 6.4 pp. Este resultado toma relevancia, pues la probabilidad de que el adolescente trabaje en un TPM se reduce si labora en el mismo sector de la madre y aumenta cuando es el mismo sector económico del padre.

En el Cuadro 6 se presentan los resultados desagregados por edad y género de los adolescentes. El coeficiente de la diferencia por género en la probabilidad de trabajar en un TPM es mayor para los adolescentes hombres de 12 a 14 años, estimada en 13.8 pp, en comparación con la estimada para los hombres adolescentes de 15 a 17 años, estimada en 11.4 pp. Para cada uno de los subgrupos que se analizan en el Cuadro 6 el coeficiente que nos muestra el impacto del ingreso mensual sobre la variable dependiente es significativo. Un aumento de mil pesos en el ingreso de los menores de 12 a 14 años incrementa en 5.4 pp la probabilidad de encontrarse en un TPM; en tanto que el efecto sería de 3.02 pp para los adolescentes de 15 a 17 años.

Al dividir por género, en el Cuadro 6, se observa que el incremento en el ingreso tiene un impacto mayor para las mujeres, ya que, si el ingreso mensual aumenta en mil pesos, la probabilidad de que las mujeres se encuentren en un TPM se incrementa en 4.2 pp; en tanto para los hombres dicha probabilidad aumenta, en promedio, $2.9 \mathrm{pp}$. Es decir, la probabilidad de insertarse en un TPM es mayor en las mujeres, en comparación de los hombres, si el salario mensual de los adolescentes aumenta.

$\mathrm{Al}$ analizar el coeficiente que nos indica que el adolescente se encuentra en el mismo sector que el padre, es mayor para los adolescentes cuyo rango de edad es de 12 a 14 años (11.8 pp), y el impacto es menor para las mujeres ( $8.5 \mathrm{pp})$. Si el adolescente labora en el mismo sector que la madre, al igual que los resultados generales del Cuadro 5 , se encuentra que la probabilidad de estar en un TPM se reduce, en mayor magnitud para los hombres, 9.88 pp, y en menor magnitud para los adolescentes de 12 a 14 años, reducción estimada en 7.5 pp y en los adolescentes de 15 a 17 años, una reducción de 5.98 pp; sin embargo, el hecho de que las mujeres adolescentes trabajen en la misma actividad de su mamá no es un factor que reduzca la probabilidad de emplearse en un TPM, pues resulta estadísticamente no significativa. El ingreso de la mamá solo es significativo en reducir la probabilidad de que un adolescente de 12 a 14 años trabaje en un TPM, pero no tiene efecto en los otros grupos considerados. Por otra parte, la escolaridad de la mamá reduce la probabilidad de que los adolescentes hombres y de los adolescentes de 15 a 17 años se inserten en TPM. Por el contrario, ni el ingreso ni la escolaridad del padre se asocian con una reducción en la probabilidad de que los adolescentes trabajen en un TPM, solo con excepción de las mujeres. 


\section{Cuadro 6}

Efectos marginales por grupos de edad y género.

\begin{tabular}{|c|c|c|c|c|}
\hline & MODELO E & MODELO F & MODELO G & MODELO H \\
\hline & 12 A 14 AÑ̃S & 15 A 17 AÑos & HOMBRES & MUJERES \\
\hline \multirow{2}{*}{ Género } & $0.138^{* * *}$ & $0.114^{* \star *}$ & ---- & ---- \\
\hline & {$[2.90]$} & {$[3.06]$} & & \\
\hline \multirow{2}{*}{ Ingreso mensual del adolescente } & $0.0000542^{* * *}$ & $0.0000302^{* * *}$ & $0.0000286^{* * *}$ & $0.0000420^{* * *}$ \\
\hline & {$[3.04]$} & {$[4.55]$} & {$[3.88]$} & {$[2.64]$} \\
\hline \multirow{2}{*}{ Edad a la que empezó a trabajar } & -0.0159 & $-0.0244^{* * *}$ & $-0.0287^{* * *}$ & 0.000305 \\
\hline & {$[-1.47]$} & {$[-4.08]$} & {$[-4.92]$} & {$[0.04]$} \\
\hline \multirow{2}{*}{ Dummy de seguro médico } & & $-0.142^{* *}$ & -0.111 & $-0.278^{* *}$ \\
\hline & & {$[-2.04]$} & {$[-1.35]$} & {$[-2.11]$} \\
\hline \multirow{2}{*}{$\begin{array}{l}\text { Dummy si adolescente trabaja en el } \\
\text { mismo sector que papá }\end{array}$} & $0.127^{\star * *}$ & $0.0962^{* * *}$ & $0.0915^{* * *}$ & $0.0849^{* *}$ \\
\hline & [2.99] & [3.73] & [3.34] & {$[2.05]$} \\
\hline \multirow{2}{*}{ Ingreso papá } & -0.00000237 & -0.00000439 & -0.00000265 & -0.00000605 \\
\hline & {$[-0.59]$} & {$[-1.63]$} & {$[-1.03]$} & {$[-1.48]$} \\
\hline \multirow{2}{*}{ Escolaridad papá } & -0.0049 & -0.00184 & -0.00426 & 0.00324 \\
\hline & {$[-0.75]$} & {$[-0.44]$} & {$[-0.99]$} & {$[0.66]$} \\
\hline \multirow{2}{*}{$\begin{array}{l}\text { Dummy si adolescente trabaja en el } \\
\text { mismo sector que mamá }\end{array}$} & $-0.0750^{*}$ & $-0.0598^{*}$ & $-0.0988^{* * *}$ & 0.0476 \\
\hline & {$[-1.71]$} & {$[-1.83]$} & {$[-3.03]$} & {$[1.12]$} \\
\hline \multirow{2}{*}{ Ingreso mamá } & $-0.000033^{* * *}$ & -0.00000424 & -0.00000511 & -0.00000958 \\
\hline & {$[-3.11]$} & {$[-1.27]$} & {$[-1.25]$} & {$[-1.42]$} \\
\hline \multirow{2}{*}{ Escolaridad mamá } & 0.00222 & $-0.0103^{* *}$ & -0.00594 & $-0.0117^{* *}$ \\
\hline & {$[0.32]$} & {$[-2.30]$} & {$[-1.31]$} & {$[-2.40]$} \\
\hline Dummies por región & Si & $\mathrm{Si}$ & Si & Si \\
\hline Constante & Si & $\mathrm{Si}$ & $\mathrm{Si}$ & $\mathrm{Si}$ \\
\hline Obs. & 14,135 & 14,168 & 14,634 & 13,667 \\
\hline Seleccionadas & 1,019 & 2,873 & 2,790 & 1,100 \\
\hline No selecc. & 13,116 & 11,295 & 11,844 & 12,567 \\
\hline Wald chi2 & 47.69 & 81.01 & 90.95 & 38.26 \\
\hline
\end{tabular}

Fuente: Elaboración propia con datos del MTI, INEGI (2017b) y Cuarto trimestre ENOE, INEGI (2017a). Nota: Significancia estadística ${ }^{\star} 90 \%,{ }^{\star *} 95 \%,{ }^{* *} 99 \%$.

En el Cuadro 7 se presentan los resultados incluyendo la tasa de riesgo del sector en el que se encuentran trabajando los padres de los adolescentes. Se espera que, si la tasa de riesgo es alta, entonces la probabilidad de que el adolescente esté en un TPM sea mayor. Algunos trabajos podrían no ser peligrosos para el padre, pero si para los hijos adolescentes, se utiliza la tasa de riesgo de los sectores de los adolescentes para clasificarlos en riesgo bajo, medio o alto. En el Cuadro 7 solo se incluyen los sectores con alta tasa de riesgo para los adolescentes, se estima un modelo general para posteriormente desagregar por grupos de edad y género. Se encuentra que en los jóvenes de 15 a 17 años no hay una diferencia significativa entre hombres y mujeres de estar en un TPM. El ingreso mensual del adolescente sigue siendo positivo, significativo y en magnitudes similares a las presentadas en los Cuadros 5 y 6 . Los 
resultados del Cuadro 7 muestran que cuando el padre se encuentra en un sector con una tasa de riesgo alta para el adolescente, el impacto de trabajar en el mismo sector que el padre incrementa la probabilidad de estar en un TPM; en mayor magnitud en comparación con los resultados obtenidos cuando se incluyen todos los adolescentes. En este caso, si el joven se encuentra en el mismo sector que el padre la probabilidad de laborar en un TPM es de 15.7 pp mayor, en comparación si trabaja en un diferente sector. En tanto que, el impacto es mayor en los adolescentes de 12 a 14 años, ya que la probabilidad de estar en un TPM se incrementa en 19.4 pp. Para los adolescentes de 15 a 17 años, el estar en el mismo sector-SCIAN que el papá, aumenta en 14.6 pp la probabilidad de participar en un TPM. Cuando se separa este subgrupo por género, se muestra que para el caso de las mujeres se encuentra un impacto mayor (17.1 pp) en comparación con los hombres (13.1 pp).

Los resultados obtenidos son consistentes con los encontrados en la literatura, ya que la ocupación del padre es relevante para el destino ocupacional del menor (Cortés y Latapí, 2005) y es de suma importancia como determinante del logro ocupacional (Zenteno y Solís, 2006); además de que hay una relación directa entre la ocupación del padre y la primera ocupación del hijo (Puga y Solís, 2010). Asimismo, los varones son más propensos a peligros en los lugares de trabajo (Dunn et al., 1998; Runyan et al., 2007). En resumen, los resultados evidencian una asociación entre el sector de actividad económica en el que trabajan el padre o la madre y en el tipo de trabajo en el que se incorpora el adolescente. Así como también otras variables de los padres son relevantes en la probabilidad de estar empleado en un TPM, tales como el ingreso y la escolaridad; siendo los hombres más propensos a estar en un trabajo peligroso en comparación con las mujeres. Además, el ingreso que percibe el adolescente es importante en la decisión de participar en un TPM, así como también si cuenta con seguro médico por parte de su trabajo. Otro de los resultados encontrados indica que entre mayor sea la edad en la que el adolescente empezó a trabajar reduce la probabilidad de encontrarse en un TPM. Por último, la influencia del sector económico es mayor del padre al hijo adolescente, en comparación con las hijas; en tanto, la influencia del sector económico de la madre reduce la probabilidad de que el hijo se incorpore en un TPM, más no tiene influencia en las hijas adolescentes.

Se realizó un ejercicio de sensibilidad de los resultados cambiando el umbral de las condiciones mínimas laborales, los resultados se muestran en el Anexo Cuadro A3. Los resultados se presentan para el corte unidimensional, $\mathrm{K}=1$, si se incumple al menos una condición laboral, y si incumple con tres dimensiones simultáneamente, $\mathrm{K}=3$. Comparando los resultados obtenidos con la medida unidimensional con los resultados presentados en el Cuadro 6, se encuentra que el efecto de encontrarse en el mismo sector que el padre es similar con ambas medidas. Solo al separar por género se aprecian diferencias: la magnitud del efecto disminuye para los hombres al usar la medida unidimensional, en tanto que para las mujeres aumenta. Por otra parte, analizando los coeficientes de la variable que indica que se encuentra en el mismo sector que la madre se identifica un mayor efecto tanto al examinar a todos los adolescentes, así como también para los hombres (al separar por género), pero el efecto se sigue diluyendo para las mujeres. Por último, al utilizar el corte de tres dimensiones, se reduce la significancia estadística de la variable dicotómica del mismo sector que la madre, en tanto que, para el padre, los resultados revelan un efecto menor tanto en el efecto general, como por género. 
Cuadro 7

Efectos marginales por grupos de edad y género.

\begin{tabular}{|c|c|c|c|c|c|}
\hline & MODELO D.1 & MODELO E.1 & MODELO F.1 & MODELO G.1 & MODELO H.1 \\
\hline & TODOS & 12 A 14 AÑos & 15 A 17 AÑOS & HOMBRES & MUJERES \\
\hline \multirow{2}{*}{ Género } & $0.105^{* *}$ & $0.163^{* *}$ & 0.0763 & ---- & ---- \\
\hline & {$[2.09]$} & {$[2.30]$} & {$[1.17]$} & & \\
\hline \multirow{2}{*}{$\begin{array}{l}\text { Ingreso mensual del } \\
\text { adolescente }\end{array}$} & $0.0000344^{* * *}$ & $0.0000730^{* * *}$ & $0.0000313^{* * *}$ & $0.0000296^{* * *}$ & $0.0000375^{*}$ \\
\hline & {$[4.00]$} & {$[2.85]$} & [3.42] & [2.99] & {$[1.66]$} \\
\hline \multirow{2}{*}{$\begin{array}{l}\text { Edad a la que empezó } \\
\text { a trabajar }\end{array}$} & $-0.0220^{* * *}$ & -0.0168 & $-0.0245^{* * *}$ & $-0.0302^{* * *}$ & $0.0173^{*}$ \\
\hline & {$[-3.24]$} & {$[-1.25]$} & {$[-3.09]$} & {$[-4.10]$} & {$[1.65]$} \\
\hline \multirow{2}{*}{$\begin{array}{l}\text { Dummy de seguro } \\
\text { médico }\end{array}$} & -0.173 & --- & -0.161 & -0.157 & -0.167 \\
\hline & {$[-1.47]$} & & {$[-1.39]$} & {$[-1.08]$} & {$[-1.17]$} \\
\hline \multirow{2}{*}{$\begin{array}{l}\text { Dummy si adolescente } \\
\text { trabaja en el mismo } \\
\text { sector que papá }\end{array}$} & $0.157^{* * *}$ & $0.194^{* * *}$ & $0.146^{\text {***}}$ & $0.131^{* * *}$ & $0.171^{*}$ \\
\hline & [4.68] & [2.97] & [3.89] & {$[3.36]$} & {$[1.74]$} \\
\hline \multirow{2}{*}{ Ingreso papá } & 0.000000475 & 0.0000073 & -0.00000238 & 0.00000394 & $-0.0000152^{*}$ \\
\hline & {$[0.14]$} & {$[1.15]$} & {$[-0.55]$} & {$[1.04]$} & {$[-1.68]$} \\
\hline \multirow{2}{*}{ Escolaridad papá } & -0.0013 & 0.00123 & -0.00253 & -0.00174 & -0.000161 \\
\hline & {$[-0.29]$} & {$[0.15]$} & {$[-0.46]$} & {$[-0.32]$} & {$[-0.03]$} \\
\hline \multirow{2}{*}{$\begin{array}{l}\text { Dummy si adolescente } \\
\text { trabaja en el mismo } \\
\text { sector que mamá }\end{array}$} & -0.0264 & -0.00453 & -0.03 & -0.0491 & 0.0413 \\
\hline & {$[-0.68]$} & {$[-0.08]$} & {$[-0.61]$} & {$[-1.15]$} & {$[0.70]$} \\
\hline \multirow{2}{*}{ Ingreso mamá } & -0.0000103 & $-0.000057^{* * *}$ & -0.00000147 & -0.0000106 & -0.0000002 \\
\hline & {$[-1.27]$} & {$[-3.00]$} & {$[-0.16]$} & {$[-1.04]$} & {$[-0.02]$} \\
\hline \multirow{2}{*}{ Escolaridad mamá } & $-0.00878^{*}$ & 0.000134 & $-0.0109^{*}$ & -0.00798 & -0.00797 \\
\hline & {$[-1.76]$} & {$[0.02]$} & {$[-1.77]$} & {$[-1.41]$} & {$[-1.31]$} \\
\hline Dummies por región & $\mathrm{Si}$ & $\mathrm{Si}$ & $\mathrm{Si}$ & $\mathrm{Si}$ & $\mathrm{Si}$ \\
\hline Constante & $\mathrm{Si}$ & $\mathrm{Si}$ & $\mathrm{Si}$ & $\mathrm{Si}$ & $\mathrm{Si}$ \\
\hline Obs. & 11,136 & 5,602 & 5,534 & 5,837 & 5,299 \\
\hline Seleccionadas & 1,972 & 543 & 1,429 & 1,523 & 449 \\
\hline No selecc. & 9,164 & 5,059 & 4,105 & 4,314 & 4,850 \\
\hline Wald chi2 & 64.24 & 35.63 & 45.28 & 54.29 & 51.95 \\
\hline
\end{tabular}

Fuente: Elaboración propia con datos del MTI, INEGI (2017b) y Cuarto trimestre ENOE, INEGI (2017a). Nota: Significancia estadística ${ }^{*} 90 \%, * * 95 \%, * * * 99 \%$.

\section{Conclusiones}

En este artículo se propuso una medida multidimensional para medir el trabajo peligroso, basada en las condiciones labores incumplidas de acuerdo con la Ley Federal del Trabajo (LFT) y en las recomendaciones de la OIT. La medida de trabajo peligroso multidimensional (TPM) representa una contribución a la literatura, ya que hasta el momento no existe una medida estándar para medir este tipo de trabajos para los trabajadores adolescentes. En términos descriptivos, se estima que en México 
el 30.53\% de los adolescentes se encuentran en TPM, una mayor proporción de hombres, 36.85\%, en comparación con las mujeres, $14.78 \%$. Se encuentran diferencias entre los estados del país, es en Chiapas en donde se estima el mayor porcentaje de adolescentes en un TPM, 61\%. Por otra parte, por sector de actividad económica, se encuentra un porcentaje similar de TPM en el sector de la construcción, 61.19\%. Cuando los adolescentes están trabajando en el mismo sector de actividad económica que el padre, la proporción de jóvenes en TPM aumenta, a 43.46\% en el caso de los hombres y 19\% en las mujeres. Cuando se condiciona a que el sector de actividad es el mismo que la mamá, el porcentaje de TPM es menor, $27.61 \%$ para los hombres y $16.58 \%$ para las mujeres.

El artículo evidencia una relación directa y significativa entre las características laborales de los padres y la probabilidad de que los adolescentes se encuentren en un TPM, por lo que, inferimos que existe un efecto intergeneracional. En términos de puntos porcentuales (pp), si el adolescente trabaja en el mismo sector que el padre aumenta la probabilidad de que esté en un TPM entre 10.3 pp y 15.7 pp. Por el contrario, la probabilidad de que el adolescente esté en un TPM, si trabajaba en el mismo sector económico de la mamá, se reduce entre 5.5 pp y 6.39 pp. Estos efectos aumentan en magnitud cuando se considera la peligrosidad y riesgo laboral de la actividad económica, más no existe efecto estadísticamente significativo de la actividad económica de la mamá.

Si los adolescentes trabajan en el mismo sector económico que su padre, el incremento en la probabilidad de estar en un TPM es mayor para el total de adolescentes de 12 a 14 años, de 12.7 pp, y el menor incremento se estima en las mujeres, $8.49 \mathrm{pp}$. Por el contrario, cuando los adolescentes están en el mismo sector de la madre, la probabilidad de estar en un TPM se reduce más para los hijos, 9.88 pp, más no se encuentra un efecto en las hijas. Al considerar el riesgo laboral del sector económico, el efecto intergeneracional del padre aumenta la magnitud del cambio, mientras que el efecto de la actividad económica de la mamá no tiene efecto sobre ningún grupo considerado.

La transmisión intergeneracional de la actividad económica del padre es el factor que más influye en la probabilidad de que los adolescentes estén en un trabajo peligroso; en tanto la ocupación de la mamá es un factor que reduce esta probabilidad, especialmente para los hombres de 15 a 17 años. En este sentido es una prioridad entender los factores que se establecen en la LFT y las recomendaciones de la OIT para erradicar el trabajo peligroso entre los menores de edad. Si bien es difícil monitorear y detectar este tipo de trabajos, los resultados de este estudio permiten inferir la influencia familiar sobre la empleabilidad de los adolescentes en trabajos en donde están expuestos a sustancias tóxicas u otros peligros, porque cargan cosas pesadas, o porque están en riesgo de sufrir alguna lesión física o mental, y en menor medida porque trabajan en lugar inapropiados para su edad o por trabajar en una jornada nocturna.

Por otra parte, la ocupación de la mamá contribuye a reducir la probabilidad de que los hijos adolescentes estén en trabajos peligrosos, pero no tiene influencia sobre la ocupación de las hijas. Este es un efecto con sesgo de género, es probable que otro problema no visualizado sea el trabajo no remunerado peligroso que podrían estar realizando, en mayor medida las mujeres, puesto que se encuentra que, si el ingreso que reciben las mujeres adolescentes aumentara, la probabilidad de estar en un trabajo peligroso remunerado aumentaría más que en el caso de los hombres. La escolaridad de la mamá reduce la probabilidad de que las hijas estén en un trabajo peligroso.

Los resultados indican que entre mayor sea el ingreso que reciben los adolescentes aumenta la probabilidad de estar en un TPM, posiblemente se encuentran en la necesidad de cubrir el ingreso mínimo que necesita el hogar para poder subsistir, y aunque la peligrosidad del empleo sea alta también reciben 
una mayor remuneración por realizarla. Por tanto, es necesaria una política encaminada a concientizar sobre los riesgos a los que están expuestos los adolescentes. Si bien el trabajo realizado por los adolescentes es un apoyo en la economía familiar, se podría transitar de un trabajo peligroso a un trabajo ligero, que a la larga erradicaría el trabajo peligroso entre los menores de edad.

\section{Referencias}

Alkire, S., y Foster, J. (2011). Counting and multidimensional poverty measurement. Journal of Public Economics, 95(7-8), 476-487. DOI: https://doi.org/10.1016/j.jpubeco.2010.11.006

Alkire, S., y Santos, M. E. (2013). A multidimensional approach: Poverty measurement \& beyond. Social Indicators Research, 112(2), 239-257. DOI: https://doi.org/10.1007/s11205-013-0257-3

Basu, Kaushik y Pham Hoang Van (1998). The economics of child labor. American Economic Review, $88(3), 412-427$.

Bourdillon, M. (2006). Children and work: A review of current literature and debates. Development and Change, 37(6), 1201-1226. DOI: https://doi.org/10.1111/j.1467-7660.2006.00519.x

Breslin, C., Koehoorn, M., Smith, P., y Manno, M. (2003). Age related differences in work injuries and permanent impairment: a comparison of workers' compensation claims among adolescents, young adults, and adults. Occupational and Environmental Medicine, 60(9), e10-e10. DOI: https://dx.doi.org/10.1136/oem.60.9.e10

Breslin, F. C., Tompa, E., Zhao, R., Amick III, B. C., Pole, J. D., Smith, P., y Hogg-Johnson, S. (2007). Work disability absence among young workers with respect to earnings losses in the following year. Scandinavian Journal of Work Environment and Health, 33(3), 192-197.

Cortés, F., y Escobar Latapí, A. (2005). Movilidad social intergeneracional en el México urbano. Revista de la CEPAL, (85), 149-167.

De Hoyos, R., Martínez de la Calle, J. M., y Székely, M. (2010). Educación y movilidad social en México. En Movilidad Social en México. Población, Desarrollo y Crecimiento (pp. 135-164). Centro de Estudios Espinosa Yglesias.

Doruk, Ö. T., Pastore, F., y Yavuz, H. (2020). Intergenerational Occupational Mobility in Latin American Economies: An Empirical Approach. IZA-Institute of Labor Economics.

Dunn, K. A., Runyan, C. W., Cohen, L. R., y Schulman, M. D. (1998). Teens at work: a statewide study of jobs, hazards, and injuries. Journal of Adolescent Health, 22(1), 19-25. DOI: https://doi. org/10.1016/S1054-139X(97)00071-2

Emerson, P. M., y Souza, A. P. (2003). Is there a child labor trap? Intergenerational persistence of child labor in Brazil. Economic Development and Cultural Change, 51(2), 375-398. DOI: https://doi. org/10.1086/346003

Evensen, C. T., Schulman, M. D., Runyan, C. W., Zakocs, R. C., y Dunn, K. A. (2000). The downside of adolescent employment: hazards and injuries among working teens in North Carolina. Journal of Adolescence, 23(5), 545-560. DOI: https://doi.org/10.1006/jado.2000.0342 
Fischer, D., Lombardi, D. A., Folkard, S., Willetts, J., y Christiani, D. C. (2017). Updating the "Risk Index": A systematic review and meta-analysis of occupational injuries and work schedule characteristics. Chronobiology International, 34(10), 1423-1438. DOI: https://doi.org/10.1080/074 20528.2017.1367305

Grajales, R. V., y Monroy-Gómez-Franco, L. Á. (2017). Movilidad social en México: hallazgos y pendientes. Revista de Economía Mexicana, (2), 97-142.

Grootaert, C., y Kanbur, R. (1995). Child labour: An economic perspective. International Labour Review, 134(2), 187.

Hard, D. L., y Myers, J. R. (2006). Fatal work-related injuries in the agriculture production sector among youth in the United States, 1992-2002. Journal of Agromedicine, 11(2), 57-65. DOI: https://doi.org/10.1300/J096v11n02_09

Heady, C. (2003). The effect of child labor on learning achievement. World Development, 31(2), 385398. DOI: https://doi.org/10.1016/S0305-750X(02)00186-9

Heckman, J. J. (1979). Sample selection bias as a specification error. Econometrica: Journal of the Econometric Society, 47(1), 153-161. DOI: https://doi.org/10.2307/1912352

INEGI (2017a). Encuesta Nacional de Ocupación y Empleo, cuarto trimestre. Instituto Nacional de Geografía y Estadística, INEGI. Disponible en: https://www.inegi.org.mx/programas/enoe/15ymas/\#Microdatos

INEGI (2017b). Módulo de Trabajo Infantil 2017, Encuesta Nacional de Ocupación y Empleo. Instituto Nacional de Estadística y Geografía. Disponible en: https://www.inegi.org.mx/programas/ $\mathrm{mti} / 2017 /$

INEGI (2018). 3.2 Millones de niños, niñas y adolescentes de 5 a 17 años trabajan en México: Módulo de Trabajo Infantil (MTI) 2017. Comunicado de Prensa Núm. 269/18. Instituto Nacional de Geografía y Estadística, INEGI. Disponible en: https://www.inegi.org.mx/contenidos/saladeprensa/ boletines/2018/EstSociodemo/MdoTrabInf2018_06.pdf

Lipscomb, H. J., y Li, L. (2001). Injuries among teens employed in the homebuilding industry in North Carolina. Injury Prevention, 7(3), 205-209. DOI: http://dx.doi.org/10.1136/ip.7.3.205

López-Calva, L. F., y Macías, A. (2010). ¿Estudias o trabajas? Deserción escolar, trabajo temprano y movilidad en México. En Movilidad Social en México. Población, Desarrollo y Crecimiento (pp. 165-187). Centro de Estudios Espinosa Yglesias.

OIT (2018). Hacia la eliminación urgente del trabajo infantil peligroso. Organización Internacional del Trabajo. Oficina Internacional del Trabajo, Servicio de Principios y derechos fundamentales en el trabajo. Disponible en: http://www.ilo.org/ipec/Informationresources/WCMS_IPEC_ PUB_30317/lang--es/index.htm

Orraca, P. (2014). El trabajo infantil en México y sus causas. Problemas del Desarrollo, 45(178), 113-137. DOI: https://doi.org/10.1016/S0301-7036(14)70878-8

Psacharopoulos, G. (1997). Child labor versus educational attainment: Some evidence from Latin America. Journal of Population Economics, 10(4), 377-386. DOI: https://doi.org/10.1007/ s001480050049

Puga, I., y Solís, P. (2010). Estratificación y transmisión de la desigualdad en Chile y México. Un estudio empírico en perspectiva comparada. En Movilidad Social en México. Población, Desarrollo y Crecimiento (pp. 189-228). Centro de Estudios Espinosa Yglesias. 
Ray, R. (2002). The determinants of child labour and child schooling in Ghana. Journal of African Economics, 11(4), 561-590. DOI: https://doi.org/10.1093/jae/11.4.561

Rohlman, D. S., Ismail, A. A., Rasoul, G. A., Bonner, M. R., Hendy, O., Mara, K., Wang, K. y Olson, J. R. (2016). A 10-month prospective study of organophosphorus pesticide exposure and neurobehavioral performance among adolescents in Egypt. Cortex, 74, 383-395. DOI: https://doi. org/10.1016/j.cortex.2015.09.011

Rosenfeld, R. A. (1978). Women's intergenerational occupational mobility. American Sociological Review, 43(1), 36-46. DOI: https://doi.org/10.2307/2094760

Runyan, C. W., Schulman, M., Dal Santo, J., Bowling, J. M., Agans, R., y Ta, M. (2007). Work-related hazards and workplace safety of US adolescents employed in the retail and service sectors. Pediatrics, 119(3), 526-534. DOI: https://doi.org/10.1542/peds.2006-2009

Secretaría del Trabajo y Previsión Social [México]. (2019). Ley Federal del Trabajo. https://www.gob. $\mathrm{mx} / \mathrm{cms} /$ uploads/attachment/file/156203/1044_Ley_Federal_del_Trabajo.pdf

Solís, P. (2018). Barreras estructurales a la movilidad social intergeneracional en México: Un enfoque multidimensional. Serie Estudios y Perspectivas, (176), 149-167.

Solon, G. (1999). Intergenerational mobility in the labor market. En Handbook of Labor Economics (Vol. 3, pp. 1761-1800). Elsevier. DOI: https://doi.org/10.1016/S1573-4463(99)03010-2

Steinberg, L. (2004). Risk taking in adolescence: what changes, and why?. Annals of the New York Academy of Sciences, 1021(1), 51-58. DOI: https://doi.org/10.1196/annals.1308.005

Sturrock, S., y Hodes, M. (2016). Child labour in low-and middle-income countries and its consequences for mental health: a systematic literature review of epidemiologic studies. European Child \& Adolescent Psychiatry, 25(12), 1273-1286. DOI: https://doi.org/10.1007/s00787-016-0864-z

Swaminathan, M. (1998). Economic growth and the persistence of child labor: Evidence from an Indian city. World Development, 26(8), 1513-1528. DOI: https://doi.org/10.1016/S0305750X(98)00063-1

Torche, F. (2010). Cambio y persistencia de la movilidad intergeneracional en México. En Movilidad Social en México. Población, Desarrollo y Crecimiento (pp. 71-134). Centro de Estudios Espinosa Yglesias.

Van de Ven, W. P., y Van Praag, B. M. (1981). The demand for deductibles in private health insurance: A probit model with sample selection. Journal of Econometrics, 17(2), 229-252. DOI: https://doi. org/10.1016/0304-4076(81)90028-2

Wahba, J. (2006). The influence of market wages and parental history on child labour and schooling in Egypt. Journal of Population Economics, 19(4), 823-852. DOI: https://doi.org/10.1007/s00148005-0014-2

Zakocs, R. C., Runyan, C. W., Schulman, M. D., Dunn, K. A., y Evensen, C. T. (1998). Improving safety for teens working in the retail trade sector: opportunities and obstacles. American Journal of Industrial Medicine, 34(4), 342-350. DOI: https://doi.org/10.1002/(SICI)1097-0274(199810)34 $: 4<342::$ AID-AJIM7>3.0.CO;2-U

Zenteno, R., y Solís, P. (2006). Continuidades y discontinuidades de la movilidad ocupacional en México. Estudios Demográficos y Urbanos, 21(3), 515-546. DOI: https://doi.org/10.24201/edu. v21i3.1241 


\section{$\mathcal{A}_{\text {nexo }}$}

\section{Cuadro A1}

Trabajo peligroso bajo distintas ponderaciones

\begin{tabular}{|l|l|l|l|l|}
\hline \multicolumn{3}{|c|}{ COMBINACIÓN DE PONDERACIONES } \\
\hline & A & B & C & D \\
\hline D1: Horario laboral & $1 / 6$ & $1 / 8$ & $1 / 5$ & $1 / 8$ \\
\hline D2: Problema de salud física & $1 / 6$ & $1 / 8$ & $1 / 10$ & $1 / 4$ \\
\hline D3: Lugar de trabajo & $1 / 6$ & $1 / 4$ & $1 / 5$ & $1 / 8$ \\
\hline D4: Carga cosas pesadas & $1 / 6$ & $1 / 8$ & $1 / 5$ & $1 / 8$ \\
\hline D5: Problema de salud mental & $1 / 6$ & $1 / 8$ & $1 / 10$ & $1 / 4$ \\
\hline D6: Peligros en lugar de trabajo & $1 / 6$ & $1 / 4$ & $1 / 5$ & $1 / 8$ \\
\hline
\end{tabular}

Porcentaje de adolescentes en TPM con criterio $\mathrm{K}=2$

\begin{tabular}{|l|l|l|l|l|}
\hline General & $30.53 \%$ & $25.65 \%$ & $21.86 \%$ & $22.54 \%$ \\
\hline Hombres & $36.85 \%$ & $31.42 \%$ & $27.27 \%$ & $26.55 \%$ \\
\hline Mujeres & $14.78 \%$ & $11.24 \%$ & $8.36 \%$ & $12.55 \%$ \\
\hline 12 a 14 años & $29.58 \%$ & $26.64 \%$ & $23.69 \%$ & $19.16 \%$ \\
\hline 15 a 17 años & $30.88 \%$ & $25.29 \%$ & $21.20 \%$ & $23.78 \%$ \\
\hline
\end{tabular}

Fuente: Elaboración propia con datos del MTI, INEGI (2017b).

Cuadro A2

Resultados de la ecuación de selección

\begin{tabular}{|c|c|c|c|c|}
\hline & MODELO A & MODELO B & MODELO C & MODELO D \\
\hline \multirow{2}{*}{ Edad del adolescente } & $0.164^{* * *}$ & $0.164^{* * *}$ & $0.164^{* * *}$ & $0.164^{* * *}$ \\
\hline & {$[18.56]$} & {$[18.46]$} & {$[18.51]$} & {$[18.45]$} \\
\hline \multirow{2}{*}{$\begin{array}{l}\text { Dummy si adolescente se encuentra } \\
\text { estudiando }\end{array}$} & $-0.977^{* * *}$ & $-0.985^{* * *}$ & $-0.983^{* * *}$ & $-0.986^{* * *}$ \\
\hline & {$[-26.59]$} & {$[-26.76]$} & {$[-26.71]$} & {$[-26.75]$} \\
\hline \multirow{2}{*}{ Genero } & $0.622^{* * *}$ & $0.622^{* * *}$ & $0.622^{* * *}$ & $0.623^{* * *}$ \\
\hline & [20.18] & {$[20.18]$} & {$[20.18]$} & [20.18] \\
\hline \multirow{2}{*}{ Ingreso papá } & -0.00000364 & -0.00000296 & -0.00000364 & -0.00000314 \\
\hline & {$[-1.40]$} & {$[-1.11]$} & {$[-1.38]$} & {$[-1.18]$} \\
\hline \multirow{2}{*}{ Escolaridad papá } & $-0.0371^{* * *}$ & $-0.0361^{* * *}$ & $-0.0374^{* * *}$ & $-0.0369^{* * *}$ \\
\hline & {$[-8.23]$} & {$[-7.81]$} & {$[-8.21]$} & {$[-7.93]$} \\
\hline \multirow{2}{*}{ Ingreso mamá } & $0.0000168^{* * *}$ & $0.0000173^{* * *}$ & $0.0000184^{* * *}$ & $0.0000182^{* * *}$ \\
\hline & [3.76] & {$[3.81]$} & [3.99] & [3.94] \\
\hline \multirow{2}{*}{ Escolaridad mamá } & $-0.0256^{* * *}$ & $-0.0254^{* * *}$ & $-0.0238^{* \star *}$ & $-0.0242^{* * *}$ \\
\hline & {$[-5.36]$} & {$[-5.24]$} & {$[-4.89]$} & {$[-4.93]$} \\
\hline
\end{tabular}

(continúa...) 


\begin{tabular}{|l|l|l|l|l|}
\hline \multicolumn{7}{|l|}{ VARIABLE DEPENDIENTE: 1 SI EL AD OLESCNTE SE ENCUENTRA TRABA A AND O; O SI NO } \\
\hline & MODELO A & MODELO B & MODELO C & MODELO D \\
\hline \multirow{3}{*}{ /athrho } & $-0.510^{* * *}$ & $-0.367^{* * *}$ & $-0.387^{* * *}$ & $-0.318^{* * *}$ \\
\cline { 2 - 6 } & {$[-7.96]$} & {$[-4.74]$} & {$[-5.13]$} & {$[-3.87]$} \\
\hline Dummies por región & $\mathrm{Si}$ & $\mathrm{Si}$ & $\mathrm{Si}$ & $\mathrm{Si}$ \\
\hline Constante & $\mathrm{Si}$ & $\mathrm{Si}$ & $\mathrm{Si}$ & $\mathrm{Si}$ \\
\hline Obs. & 28301 & 28301 & 28301 & 28301 \\
\hline
\end{tabular}

Fuente: Elaboración propia con datos del MTI, INEGI (2017b) y Cuarto trimestre ENOE, INEGI (2017a). Nota: Significancia estadística ${ }^{\star} 90 \%,{ }^{* *} 95 \%,{ }^{* *} 99 \%$.

Cuadro A3
Efectos marginales utilizando diferentes cortes, $\mathrm{K}=1, \mathrm{~K}=2$ y $\mathrm{K}=3$

\begin{tabular}{|c|c|c|c|}
\hline & $\begin{array}{l}\text { USANDO MEDIDA } \\
\text { UNIDIMENSIONAL, K=1 } \\
\text { MODELO A3.1 }\end{array}$ & $\begin{array}{l}\text { USANDO MEDIDA DE } \\
\text { CORTE, } K=2 \\
\text { MODELO D }\end{array}$ & $\begin{array}{l}\text { USANDO MEDIDA DE } \\
\text { CORTE, K=3 } \\
\text { MODELO A3.2 }\end{array}$ \\
\hline \multirow{2}{*}{ Ingreso mensual del adolescente } & $0.00003^{* * *}$ & $0.00003^{* * *}$ & $0.00003^{* * *}$ \\
\hline & [4.63] & {$[5.30]$} & {$[5.14]$} \\
\hline \multirow{2}{*}{$\begin{array}{l}\text { Dummy si adolescente trabaja en el } \\
\text { mismo sector que papá }\end{array}$} & $0.0919^{* * *}$ & $0.103^{* * *}$ & $0.0596^{* * *}$ \\
\hline & [4.75] & [4.61] & [2.71] \\
\hline \multirow{2}{*}{ Ingreso papá } & -0.000002 & $-0.00000381^{*}$ & $-0.00001^{* *}$ \\
\hline & {$[-1.02]$} & {$[-1.68]$} & {$[-2.49]$} \\
\hline \multirow{2}{*}{ Escolaridad papá } & -0.000841 & -0.00261 & -0.00215 \\
\hline & {$[-0.29]$} & {$[-0.74]$} & {$[-0.65]$} \\
\hline \multirow{2}{*}{$\begin{array}{l}\text { Dummy si adolescente trabaja en el } \\
\text { mismo sector que mamá }\end{array}$} & $-0.10^{* * *}$ & $-0.0639^{* *}$ & -0.00456 \\
\hline & {$[-4.65]$} & {$[-2.40]$} & {$[-0.17]$} \\
\hline \multirow{2}{*}{ Ingreso mamá } & 0.00000191 & $-0.00000774^{* *}$ & -0.000002 \\
\hline & {$[0.67]$} & {$[-2.29]$} & {$[-0.63]$} \\
\hline \multirow{2}{*}{ Escolaridad mamá } & $-0.0078^{* *}$ & $-0.00788^{* *}$ & $-0.00593^{*}$ \\
\hline & {$[-2.42]$} & {$[-2.11]$} & {$[-1.67]$} \\
\hline Otras variables & $\mathrm{Si}$ & $\mathrm{Si}$ & $\mathrm{Si}$ \\
\hline
\end{tabular}

Fuente: Elaboración propia con datos del MTI, INEGI (2017b) y Cuarto trimestre ENOE, INEGI (2017a). Nota: Significancia estadística ${ }^{\star} 90 \%,{ }^{*} 95 \%,{ }^{* *} 99 \%$. 